\title{
Geometric Discrete Unified Theory Framework
}

\author{
Yan Breek \\ Dedicated to my physics and math teachers Vadim Knizhnik, Boris Dubrovin, \\ Sergei P. Novikov, and Sergei Nesterov.
}

\begin{abstract}
This article proposes a unified theory framework encompassing a discrete topological interpretation of physical forces, wave functions, and the nature of space and time. It provides novel explanations for the collapse of wave functions, quantum entanglement, and offers insights into the origins of quantum probabilities. This article also explains the nature of mass, Higgs field, and suggests a path for unifying quantum mechanics and gravity. Elementary particles are represented as defects in discrete topological spaces. Entangled particles are directly connected to each other through a puncture in discrete space, separated by a distance of one Planck length. Wave functions are explained as mechanical stress waves within elastic discrete space. The results of the double-slit experiment are interpreted as wave functions maximizing the probability of rupture in high-stress areas of discrete space with obvious analogies to solid state mechanics. Wave-particle duality is explained as discrete topological defects causing extended distributed stress within space lattice.
\end{abstract}

\section{Introduction}

A large portion of the work in theoretical physics currently focuses more on the mathematical formulas rather than the underlying conceptual framework. It has long been accepted that the conceptual foundation of quantum mechanics is not as consistent and appealing as the elegant framework of general relativity. For instance, as was pointed out by Landau et al. [1], although quantum mechanics describes quantum phenomena, the concept of a "measurement," which is fundamental to quantum mechanics, is a classical idea, which is a 
foundational inconsistency. It has also proved very difficult to unify gravity with quantum mechanics. The lack of coherence and consistency in quantum mechanics is partially due to the fact that, while general relativity was developed solely by Einstein based on a powerful physical insight, quantum mechanics was developed piece-wise by multiple physicists based more on mathematical formulae than on underlying physical principles and insights.

Although mathematics is a crucial tool for expressing and developing ideas in theoretical physics, it is not the only approach. For example, the original scientific articles written by Michael Faraday contained little to no math but, nevertheless, introduced novel and useful physical concepts of magnetic fields and the behavior of magnetic field lines that later were developed into part of a powerful mathematical framework by James Maxwell. Einstein's "happiest thought" was the physical equivalence principle. Even Feynman diagrams were and are popular due to their simple and intuitive geometric interpretation. While physics has progressed tremendously since Faraday and Einstein, their physical insight-based approach, which expresses ideas in geometric or topological terms, remains valid and provides a fruitful foundation on which to develop the mathematical framework.

In this paper, I propose several concepts and insights that may lay out a foundation of a unified theory of all fundamental physical forces, which unifies gravity with quantum mechanics. While the ideas described here are not presented in a rigorous mathematical framework, I hope they can provide a foundation for one.

\section{Conceptual Foundation of the Theory: Basic postulates and assumptions}

Since the theory presented here is essentially geometric in nature and Euclidian geometry is based on axioms, I have similarly formulated the basic assumptions of the theory as a set of postulates. ${ }^{1}$

\footnotetext{
${ }^{1}$ Why Postulates? The spirit of Hilbert's 6th problem, as originally formulated, calls for creating an axiomatic system of theoretical physics. Depending on the interpretation, this problem has been later considered by most researchers as not being formulated sufficiently precise to be meaningful, especially in the light of physics discoveries of the 20th century, or perhaps not solvable at all. However, if one believes that our universe can be described as a discrete graph with rule-based operations defined on it, as proposed in this article, then perhaps the 6th Hilbert's problem starts to look as potentially tractable and solvable. Another reason is that by slightly modifying postulates one can progress to more advanced or more correct versions of this framework, just like modifying Euclid's fifth postulate led to the expansion of Euclidean geometry into Riemannian geometry. A similar approach may be applied to the proposed framework.
} 
(a)

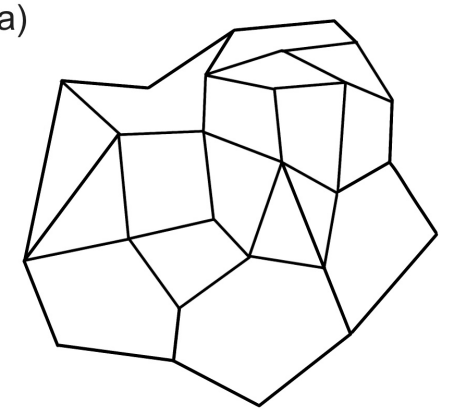

(c)

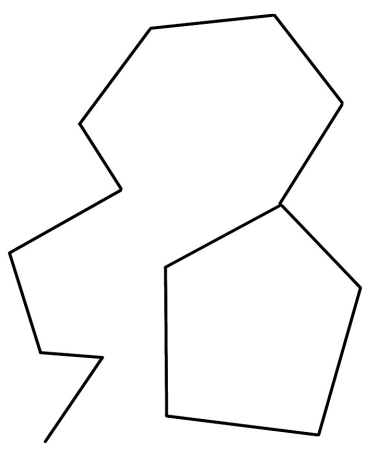

(b)

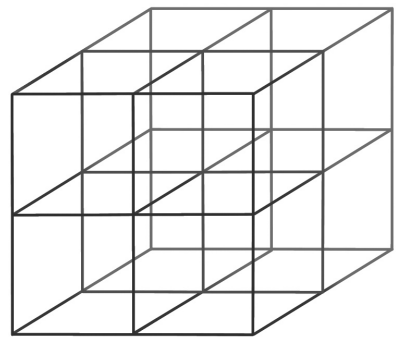

(d)

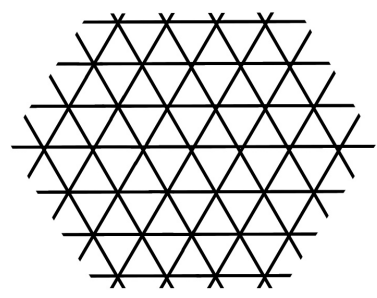

FiguRE 1. Examples of graphs and lattices, some of which may be used to model space structure in different Universes. A: A well-connected graph, no regular structure, distance can be defined, can be mapped to "wrinkled 2D" space but is not 2D in a Erdös-Harary-Tutte Euclidean unit graph sense. Unlikely to correspond to any real Universe because of irregularity. B: A well-connected lattice with 3 dimensions, regular symmetry structure for discrete translations and rotations, distance easily defined. Resembles our current Universe in many but likely not all aspects. C: Weakly connected graph, no regular structure, distance may be defined, local dimensionality 1 in most nodes, global dimensionality 2 in Erdös-Harary-Tutte $\operatorname{dim}(G)$ Euclidean unit graph sense. This graph is highly unlikely to represent any real Universe because it is way too simple and small. D: Well-connected graph, 2 dimensions, regular triangular symmetry structure for discrete translations and rotations, distance easily defined. Does not match our Universe because the number of dimensions is too small. 
Postulate 1. (Discreteness): Space is discrete and composed of the underlying elementary units. The resulting discrete structure can be geometrically represented as a graph, network, or lattice (see Figure 1). The graph does not exist in space; rather, the graph itself is space. 2

Physical Justification: The existence of fundamental constants 'Planck distance' and 'Planck time', combined with the fact that most of the physical quantities in a bound system on a quantum scale, such as energy, momentum, etc., come in discrete units (quants) suggest that space and time may likewise be discrete. This concept is supported by multiple researchers in the field of quantum gravity, such as Roger Penrose and Lee Smolin. [2-4] I assume that all of the graph's edges are on the order of one Plank length in size. ${ }^{3}$

Postulate 2. (Emergence of space): Space, as we know it, is an emergent phenomenon that resulted from the underlying elementary units connecting together in a graph with certain geometric and topological structure and properties. The local structure and properties of the space graph may vary between different universes or even between different parts of the same universe. They may also change within the same Universe over time. Space graph structure is the cause of concepts of locality, distance, dimensionality, smoothness, regularity, and symmetry; they are all emergent properties of the graph.

Physical Justification: The Big Bang was a violent dynamic process, during the course of which space appeared to be created, and time appeared to come into existence. Therefore, any truly fundamental theory must explain the nature and dynamic of space-time creation, particularly on the quantum scale (Planck length scale). A fundamental theory also has to explain what space is and what time is. As argued by Lee Smolin and Roger Penrose, it is not sufficient to merely assume spacetime as a background, like most physics theories do. $[\mathbf{2}, \mathbf{4}]$

It appears likely that the spatial structure and properties inside a black hole are different from the spatial structure and properties

\footnotetext{
${ }^{2}$ When speaking about dimensionality, I need to mention that there are several alternative ways to define dimensionality on a graph. It is also useful to make a distinction between local and global dimensionality, and between graph dimensionality and continuous space dimensionality. In some definitions of dimensionality, the number of dimensions may even be fractional. Ultimately, what is important is the underlying structure of the graph as it represents the fundamental underlying physical reality; dimensionality is a derived property.

${ }^{3}$ While referring to elements of the space graph, I will use the words "arc" and "edge" interchangeably; I will also use the words "node" and "vertex" interchangeably. I will also use the words "graph" and "lattice" interchangeably."
} 


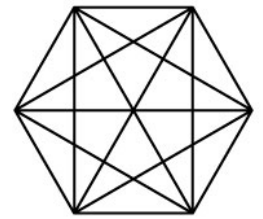

(a)

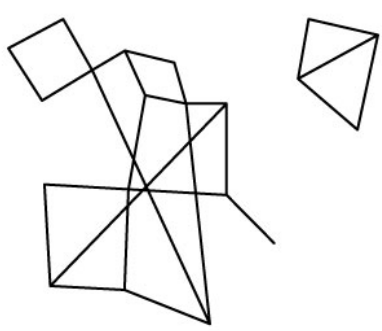

(b)

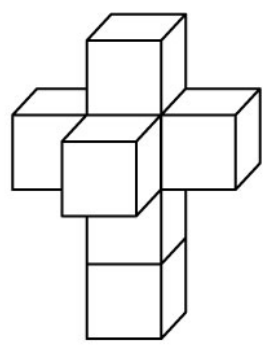

(c)

Figure 2. More examples of graphs, some of which may be used to model different Universes. A: Everything is connected with everything. This type of graph may represent our Universe just before Big Bang, because it is highly symmetrical, possibly causing unification of fundamental forces due to high symmetry. B: Highly irregular disconnected graph. That graph likely does not represent any physical universe due to it's extreme irregularity, small size, and the presence of completely disconnected areas. C: This is a 3D unfolding of a 4D cube. This graph probably does not map to any physical Universe, but it illustrates a concept of how a surface (3D) in high-dimensional space (4D) may be projected into a lower-dimensional space (3D)

outside of it. Nothing can escape from inside a black hole, and no object can move away from the center of a black hole while inside the event horizon, but outside of a black hole a physical object can move in any direction. This suggests different symmetry properties and dynamics of space inside and outside of a black hole, representing a phase transition of the space' microscopic structure on the Planck scale. Likely, the same principle applies to the Big Bang, especially if one assumes, as has been proposed by multiple authors, that the Big Bang was what emerged on the other side of a black hole, or that Big Bang is a "black hole played backward." [5-12] For these reasons, the properties and structure of space must be a changing, evolving phenomenon. This approach is inspired by multiple quantum gravity theories given by Roger Penrose, Lee Smolin, and others. $[\mathbf{2}, \mathbf{4}]$

The emergence of space is similar to how Lego blocks may be used to build a toy house or an airplane. If the elementary units had been connected differently, we could have ended up with a different number of dimensions in our universe, or perhaps with no dimensions, no symmetry, no regularity, or no locality at all (see Figure 2).

General relativity says that the curvature of space is controlled by mass and is changing over time and space. I propose to take it further and say that not only does space curvature change, but the 
(a)

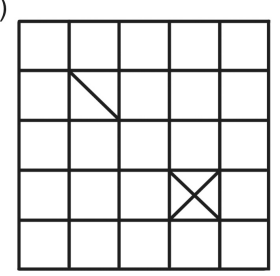

(b)

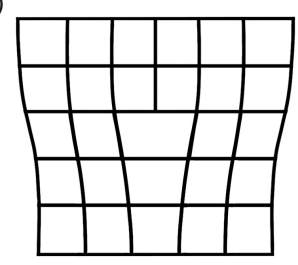

(c)

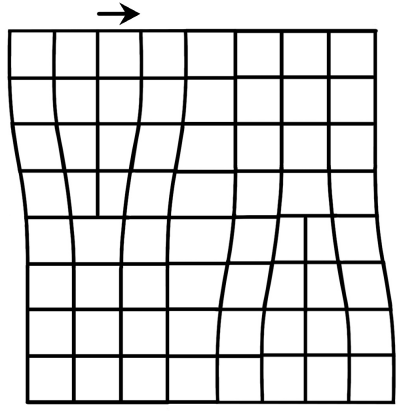

Figure 3. Topological defects representing elementary particles, and their interactions. A: Examples of two topological defects on a regular lattice. The defects represent two different possible types of elementary particles. B: 2D examples of "edge dislocations" representing possible elementary particles. The drawing also illustrates elastic stress within the graph introduced by defects (see Postulate 5 below for details). Edge dislocations also frequently occur in solid state crystals in 3D in our world. C: 2D example of two "edge dislocations" of opposite signs representing a particle and an antiparticle. The drawing also illustrate elastic/mechanical stress in the graph caused by those defects (see Postulate 5). In this configuration the stress would cause the dislocations to attract to each other and annihilate. Such interactions indeed occur in solid state crystals in our world in $3 \mathrm{D}$, especially in metals.

discrete structure, symmetry, and dimensionality of space also change, and they do so on both a quantum (Planck length) and macro scale. At the moment, I make no assumptions about the specific structure and group symmetry of the space graph on a Planck scale in our universe but will discuss that important issue in more detail below. ${ }^{4}$

Postulate 3. (Elementary particles, space defects, and quantum numbers): All elementary particles in our universe are topological defects in the underlying space lattice. Each distinct type of topological defect corresponds to a specific type of elementary particle (i.e., a defect of a certain topological structure represents an electron; a different type of defect represents a graviton, etc.) (see Figure 3).

\footnotetext{
${ }^{4}$ It is possible that Figure 2A corresponds to the state of the universe just before the Big Bang, representing a higher degree of symmetry and a compact fully connected and tightly packed space, and perhaps snapping of one edge caused the Big Bang to unravel, similar to the proposal by Cumrun Vafa and Robert Brandenberger for an ensemble of tightly wound strings some of which have later annihilated and caused the Big Bang to unravel. [13]
} 
Corollary: I further assert that all of the quantum numbers currently utilized in quantum physics are, in fact, geometric and topological characteristics of defects in the space graph that correspond to the particle or system being considered. This goes to the heart of the origin of quanta and discreteness in quantum mechanics, electric charge and spin, flavor quantum numbers, and other fundamental constants and quantum variables.

Clarification: Since it only makes sense to speak about "defects" if the underlying graph itself has certain regularity, I therefore have to assume that the space graph is indeed regular in a certain sense at least over short distances. While I do not claim to know the exact structure of that regularity, I will discuss several possibilities below which will be a focus of further research.

Physical Justification: The discrete lattice approach has been used very productively in solid state physics to describe material structure and various geometric defects and perturbations in a crystalline lattice, such as edge and screw dislocations in metals, point defects, holes, phonons, etc. A lattice has a clear and compelling topological and physical interpretation. It is appealing to describe various quantum numbers associated with elementary particles simply as topological invariants and/or graph invariants of specific types of defects in the underlying lattice. It is even more appealing that this proposal reduces the complexity and the number of assumptions in the theory. In the existing theories, space and particles are postulated and considered separately. In the proposed framework, particles are just an emergent property of the space itself.

By the way of analogy, consider what happens when two opposite but aligned edge dislocations meet in metal (see Figure 3C). Dislocations can move around, as indicated by the arrows in Figure 3C. When the top dislocation meets the fully aligned bottom dislocation, they annihilate, elastic stress in the underlying lattice is released, and phonons (vibrations of the lattice) are produced. ${ }^{5}$

\footnotetext{
${ }^{5}$ That process is known in metallurgy and solid state physics as annealing. In metallurgy, it refers to heating metals in order to force dislocations to move around more, to interact with each other, and to disappear either through annihilation or by moving to the surface, releasing the internal mechanical stress of the material in the process. In semiconductor manufacturing, it refers to heating semiconductor materials to move dopants from interstitial to substitutional lattice sites and to recrystallize amorphous areas; these processes also reduce mechanical stress and increase order within the material.
} 
I propose that this is exactly what happens when elementary particles annihilate in our universe. I also propose that this exactly represents the difference between particles and antiparticles. They are geometrically and topologically opposites of each other (in relation to the undisturbed space graph state) and can therefore geometrically and topologically annihilate each other within the space graph structure. In fact, this explains the CP symmetry - particles and antiparticles are indeed topological opposites of each other in relation to the undisturbed space lattice, and electric charge is one of the geometric/topological characteristics of the space lattice defect representing a particle.

This also raises an interesting possibility that perhaps strings are one-dimensional dislocations in the underlying structure of the space graph, or more generalized versions of dislocations (possibly including branes, etc.). Perhaps, a screw-like dislocation of the space graph represents one type of string, an edge-like dislocation represents another type, and so on. If true, then perhaps the much sought-after M-theory of strings is actually a generalized theory of various types of extended topological defects within the space graph (for another interpretation of M-theory, please see the Interpretation of Strings section below).

I will discuss below the issue of whether or not strings physically exist. I do not take a firm position at the moment on the physical reality of strings, but I do assert that, if strings do exist, they are not a fundamental but rather an emerging phenomena that owes its existence to the nature of the underlying space graph proposed in this article. It is also possible that strings are merely arbitrarily drawn imaginary contours on the space graph and therefore are a calculation trick rather than a physical object (see more discussion below). If indeed they are imaginary contours, then the only thing that is "real" about them is the fact that they must be arbitrarily selected along the arcs of the space graph (similar to the discrete elements method utilized in static mechanics and civil engineering).

Quantum numbers discussion: If elementary particles are indeed topological defects in a discrete graph structure of space, interpreting particles' internal quantum numbers (electric charge, absolute spin, etc.) as topological invariants of such defects appears to be a natural consequence. As to exactly how to map a specific quantum number to the geometric properties of the space graph defect, while I do not have a complete answer, I can provide a few illustrations. Consider, for instance, a regular space graph where each node has exactly $\mathrm{K}$ arcs connected to it. In that scenario, one of possible quantum numbers for a space graph defect may be the difference between the actual 
(a)

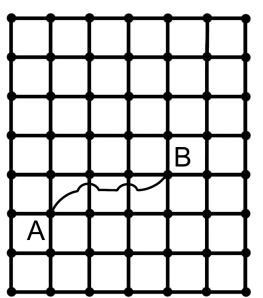

(b)

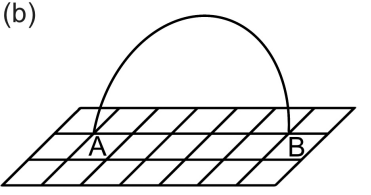

(c)

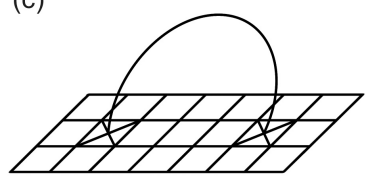

Figure 4. Direct spatial links of one Planck length between seemingly distant nodes on a regular lattice/graph. These images illustrate the nature of quantum entanglement. A, B: Direct links between seemingly distant nodes are depicted. This is a Riemann space puncture on discrete lattice/graph. 4C:This image illustrates and explains the true nature of entangled particles. Two topological defects representing elementary particles are directly connected by a direct spatial link of one Planck length. This explains "spooky action at a distance", Bell theorem, and other non-local paradoxes of quantum mechanics. Seemingly non-local action becomes local due to a presence of direct spatial link, equivalent of Riemann surface puncture on a lattice.

number of the arcs connected to the "defective" node and K. Alternatively, a quantum number may be the difference between the actual number of arcs within an elementary space graph cell representing the graph defect and the number of arcs within a "normal" space graph cell that contains no defects. Yet another quantum number may indicate the differences between the actual and the baseline number of nodes within an elementary cell. That example may refer to scalar quantum number such as electric charge, for example. The illustration in Figure 7 indicates that static electric field and electric charge may correspond to a topological defect of having either an interstitial node or a vacancy, representing the electric charges of opposite signs. See more detailed description of that below. Yet more complex quantum numbers would emerge when one considers the geometric aspects of the relationship between the defect and the local geometry of the graph. I would then perhaps expect vector-like quantities to emerge, such as spin, etc.

Postulate 4. (Non-locality or quasi-locality). Any elementary unit (A) may potentially be directly connected by an arc to any other elementary unit (B) (subject to certain conservation rules), no matter how many steps along the arcs of the regular space graph lattice separate the units $A$ and B. See Figure 4. 

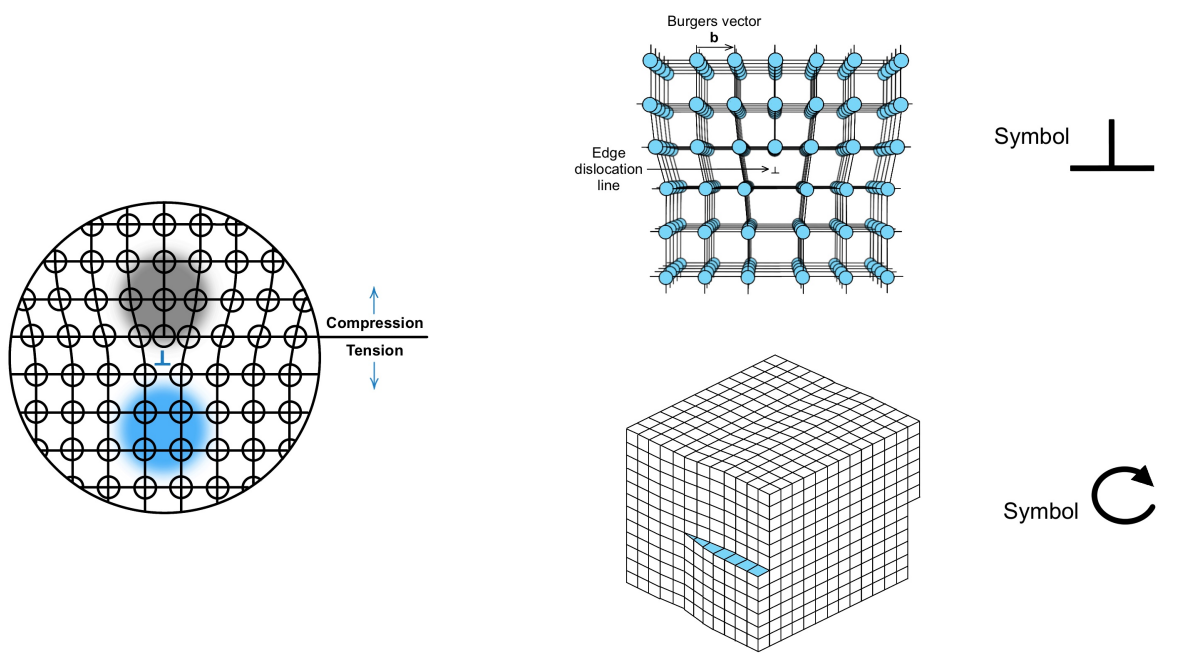

Figure 5. Edge dislocation (top right) and screw dislocation (bottom right) that occur in crystals, especially metals. Image on the left illustrates elastic stress field of an edge dislocation, explaining how topological defects in elastic structures may cause elastic stress force field to come into existence. I propose that similar mechanism is responsible for the existence of elementary particles and fundamental forces in our Universe: particles are topological defects in the space lattice. These defects cause elastic stress fields of various configurations. Those stress fields manifest themselves as four fundamental forces in our world. Images on the right illustrate two different types of linear dislocations (defects) in solid crystals. Perhaps those linear defects explain the nature of strings in string theories.

Physical Justification: These connections beautifully explain quantum entanglement, as well as other quantum phenomena that appear to violate the concept of locality. I assert that the nature of quantum entanglement is exactly represented in Figure 4C. The paradox of entangled particles is explained here simply by assuming that entangled particles are directly connected with each other and that the distance between them is one Planck length no matter how far they appear from each other. Therefore, the "spooky action at a distance" that bothered Einstein so much can be explained as a local action! See detailed discussion below regarding that important insight.

Postulate 5. (Lattice distortion, elastic stress, and the wave function): Any topological defect (elementary particle) in the space lattice results in long-range distortions and mechanical stress in the lattice. This distributed stress is mathematically represented by the wave function as described by the Schrodinger equation. 


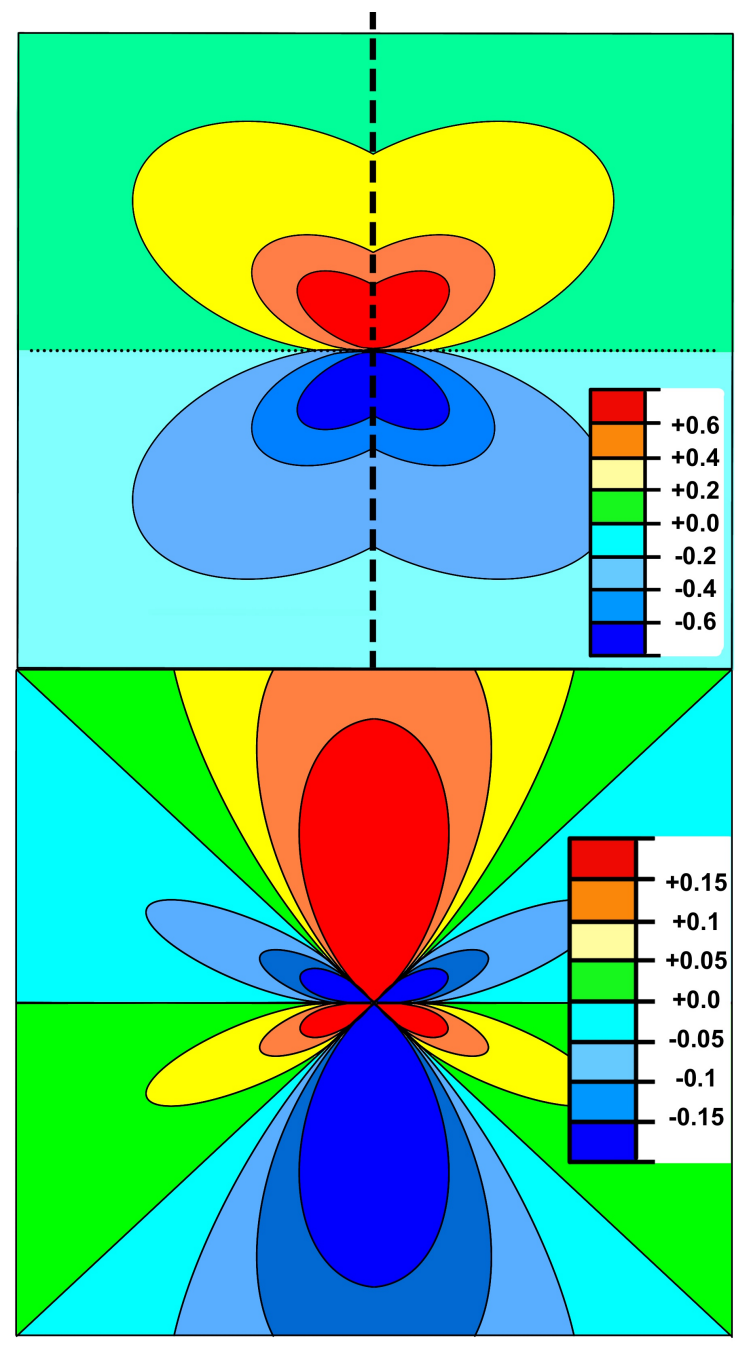

Figure 6. Mechanical stress field components ( $\mathrm{xx}$ and yy) of an edge dislocation in a crystal. The bottom image has an uncanny resemblance to certain electron orbitals of a Hydrogen atom. That resemblance may be suggesting that the proposed theory is on the right track: I propose that wave function is a degree of elastic stress caused by geometric defects in discrete space lattice. Since the stress field of a simple linear dislocation depicted here resembles electron's wave function near a simple atom, that gives us a confirmation that the proposed framework can indeed produce solutions that resemble our Universe.

Physical Justification: This is similar to the way dislocations, point defects, and other defects in crystals result in long-range distortions and a mechanical (elastic) stress field that both diminish gradually with distance. The physical nature of the wave function is the degree of mechanical stress in the space lattice/graph. See Figure $5^{6}$ and

\footnotetext{
${ }^{6}$ Figure 5 image credit: http://academic.uprm.edu/pcaceres/Courses/MechMet/MET-
} 4A.pdf 

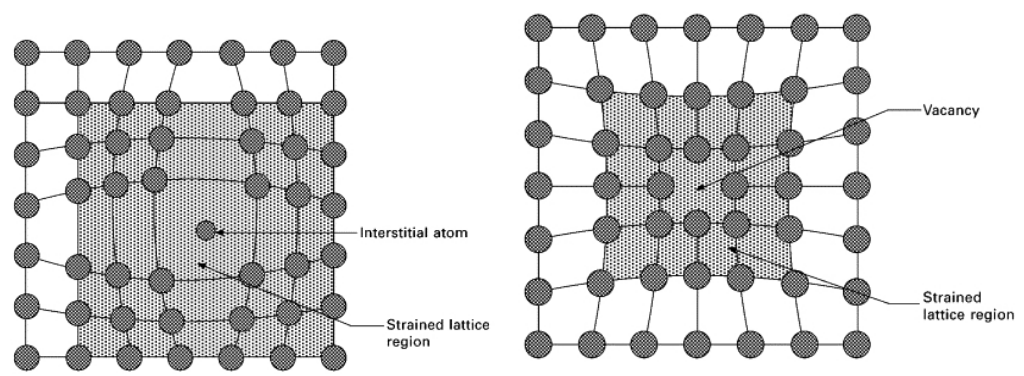

Figure 7. Elastic stress fields of point defects in crystals. They resemble the fields of positive and negative electric charges, and they interact with each other similarly: stress fields would cause an interstitial atom to be attracted to a vacancy, just like a positive charge would attract to an electric charge. An interstitial atom and a vacancy may also annihilate. These images therefore may illustrate a true nature of electrically charged particles and their oppositely charged antiparticles within the space lattice. A: Stress field of an interstitial atom in a crystal. B:: Stress field of a vacancy in a crystal.

Figure $6^{7}$ for illustrations of mechanical stress fields of linear defects, and Figure $7^{8}$ for illustrations of stress fields of point defects. Since the graph itself is space (as opposed to "existing in space"), when I speak about "stress," I mean stress in the force and energy sense. Formally, stress is a number that is assigned to each arc (and/or possibly to each node) of the graph. Our working hypothesis is that the quantitative nature of that stress is elastic, causing harmonic oscillators to appear in many equations of modern theoretical physics on the quantum level. If strings are indeed contours on the graph, this would also explain the elastic nature of strings.

Stress fields, illustrated in Figure 7, resemble corresponding electric fields of positive and negative electric charges. It is plausible that they indeed represent the nature and origin of positive and negative electric charges (an extra node causing compression, a missing node causing tension). If so, that would also explain why electric charges of the opposite sign would attract each other and why charges of the same sign would repel.

This postulate provides a highly compelling explanation of the nature of the wave function, and, in particular, wave function collapse and wave-particle duality. Wave-particle duality is resolved by associating the particle part of the duality with the corresponding discrete

\footnotetext{
${ }^{7}$ Figure 6 image credit: https://www.slideshare.net/vamsikrishna393950/stressfields-around-dislocation

${ }^{8}$ Figure 7 image credit/copyright: https://www.sciencedirect.com/topics/chemistry/crystaldisplacement
} 
topological defect, and the wave part with the resulting spatially distributed wave-like elastic stress. It also follows Einstein's tradition of literally interpreting any fundamental mathematical quantity in physics equations, including the wave function, as physically existing. This approach is also similar to pilot-wave theory of de Broglie and Bohm. The geometric interpretation proposed here is attractive and self-consistent: if an elementary particle is a topological defect in the regular elastic structure of space, such a defect must cause elastic stress in the lattice! In this representation, the particle and the wave characteristics of an elementary particle are intrinsically and unavoidably coupled with each other, as confirmed by numerous experiments.

Furthermore, sometimes high mechanical stress (or a high gradient of stress) may force a particular arc (edge) to snap and form a connection with a different node, resulting in a change in the local configuration of the graph, local energy decrease and stress relaxation, and an increase in entropy. This would explain the paradox of the dual slit experiment. See below for a detailed discussion of that insight.

\subsection{Additional Postulates (dynamic).}

Postulate 6. (Nature of time). An elementary unit of local time (Planck time) corresponds to one elementary unit of change in the underlying graph.

Clarification: One of the simplest units of change is a switch of an end of a graph's arc from node A to node B (see Figure 8A). Time is always a local phenomenon, just as in special and general relativity.

Other possible elementary switches may include the breaking of an arc (Figure 8B) or the appearance of a new arc (Figure $8 \mathrm{C}$ ), subject to appropriate conservation laws (see more on conservation laws below). In fact, an elementary switch operation (Figure 8A) can be represented as a superposition of the elementary operations (Figure 8B) and (Figure $8 \mathrm{C}$ ), as illustrated in Figure 8. This is equivalent to the annihilation operator (a-) (result depicted in Figure 8B) and the creation operator $(\mathrm{a}+)$ (Figure 8C), which are commonly utilized in quantum mechanics. Another possible elementary unit of change is an appearance of a new node or the disappearance of an existing one, or, perhaps more likely, a pairwise combination of both, if an appropriate nodes number conservation law applies (see more on that below). ${ }^{9}$

\footnotetext{
${ }^{9}$ The pairwise option (arc plus arc hole, or extra node plus node hole) may also describe the pairs of virtual particles and antiparticles that are known to spontaneously flicker in and out of existence in a vacuum.
} 


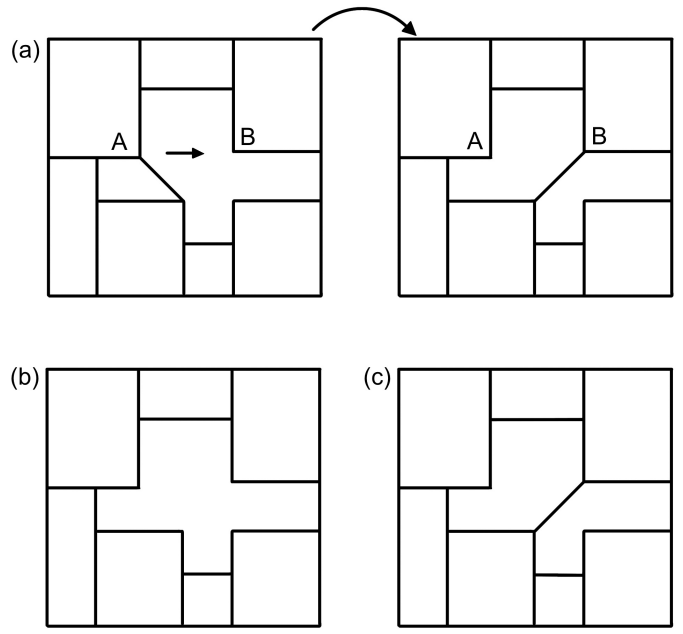

FigURE 8. Elementary switch operations on space graph, representing local passage of time. Local time is defined as the number of elementary switch operations ("clock ticks") on the space lattice. A: One-step elementary switch operation within the space lattice (also one local clock tick). B,C: Results of an 'arc annihilation' operation (B) and 'arc creation' operation $(\mathrm{C})$, that in combination result in one elementary switch operation (A) (one local clock tick).

Physical Justification: This is a natural interpretation of time given the first five postulates. Time is generally thought of as a measure of change, and, if space is discrete at Planck's scale, then the smallest possible unit of time must correspond to the smallest possible unit of change in that graph (lattice).

One may question whether or not the operation illustrated in (Figure $8 \mathrm{~A}$ should be thought of as elementary given the fact that it can be represented as a linear combination (superposition) of (Figure $8 \mathrm{~B}$ and Figure 8C). One possibility, depending on the interpretation, is that this may merely be a matter of semantics. Another possibility, that is physical rather than semantic in nature, is that, if a conservation law prohibits an operation given in (Figure 8B) from occurring alone and only allows it to occur in combination with an operation depicted in (Figure 8C) (e.g., a conservation law which preserves the total number of arcs), then (Figure 8A) can be viewed as the most elementary operation allowed by nature.

Please note that at the moment I refrain from declaring a definite form(s) for the specific conservation laws in the space graph but will discuss several possibilities later in this article. 
Since on macro scale in the classical limit the time advances smoothly and uniformly, and since according to Postulate 6 local time is a count of local elementary switches, we have to assume that the underlying space graph is constantly undergoing a sequence of elementary switches in every location throughout its structure. Since we also assert that elementary particles are geometric defects in the space graph, it follows that therefore virtual particles will be constantly created and annihilated by those elementary switch operations, producing vacuum fluctuations of vacuum energy, and creating quantum foam that was first proposed by John Wheeler [14]. Therefore, the passage of time, quantum foam, and creation and annihilation of virtual particles are all caused by and/or refer to the same underlying physical phenomena: ongoing elementary switch operations occurring throughout the space graph. One can think of them as discrete oscillators or clocks operating in every point of the discrete space graph ${ }^{10}$

Postulate 7. (Quantum probabilities). The probabilities inherent in quantum mechanics result from the elastic stress and the underlying discrete structural symmetry of the space lattice

Physical Justification: The arcs (units) of the space graph with a high amount of stress concentrated in them are more likely to snap or change structure, producing a corresponding increase of entropy due to achieving a more uniform state. By the way of analogy, this is similar to bending an irregularly shaped stick by holding it at both ends and applying force. The stick may break in any location, but it is most likely to break in the area with the highest stress. This explains why the probability of finding a particle in a specific location is proportional to the square of wave function amplitude. This is also somewhat similar to how a metal structure with large number of defects (for instance, created during the process of rapid transition from liquid to solid phase) gradually relaxes the internal mechanical stress by recombining and annihilating the defects, particularly when heated, a process that is called "annealing" in metallurgy and solid state physics.

\footnotetext{
${ }^{10}$ It is also likely that the elementary switch operations described here are topological generalizations of the cellular automata operations, as described by Stephen Wolfram and others in various publications [15]. The only differences are that, in the case of the space graph, operations occur on the graph and are additionally quantified by various intrinsic quantities (such as stress) and probabilities (see more on probabilities below). Perhaps, an extended topologically generalized theory of cellular automata operations may prove very useful for modeling this. Some papers have been already published in that field [16], although it appears that some of those papers assume Euclidean geometry, a condition that should be relaxed to adequately model space as part of a geometric discrete unified field theory.
} 
(a)

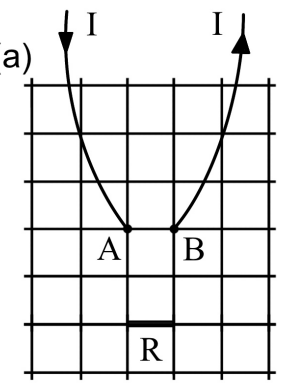

(b)

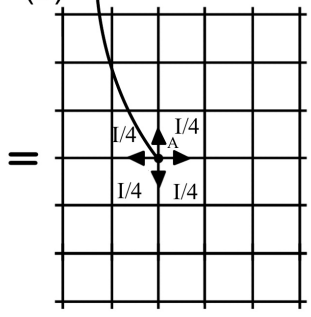

(c)

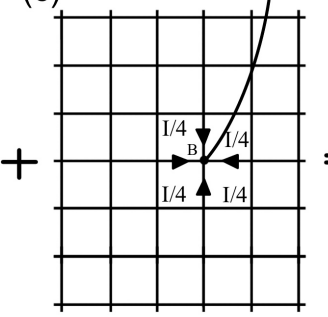

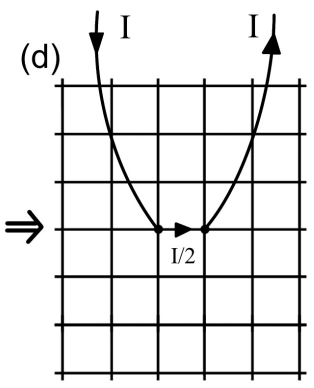

FIGURE 9. Illustration of solving currents flow equations on infinite conducting lattice based on symmetry considerations. Similar approach may be applicable for calculating elementary particles paths' and measurement probabilities on the space graph (lattice) based on symmetry structure and elastic stress within each arc. In this analogy electric resistance within the conducting network is analogous to elastic stress in the space graph; symmetry considerations would apply equally to the conducting network and to the space lattice. A: Problem setting: calculate the entire lattice' electric resistance for the depicted configuration. B: Lattice symmetry results in inflow current I splitting equally into I/4 currents in each of the four adjacent arcs. C: Outflow currents are also symmetrical, each of them equal to I/4. D: Due to Kirchhoff's laws, configuration in $\mathrm{A}$ is equal to a superposition of $\mathrm{B}$ and $\mathrm{C}$. Therefore the current from point $\mathrm{A}$ to point $\mathrm{B}$ must be equal to $\mathrm{I} / 4+\mathrm{I} / 4=\mathrm{I} / 2$. Therefore total resistance is $\mathrm{R} / 2$.

Also, when a unit of change travels along a certain path and encounters a split in the graph (say, left or right), the probability of the unit taking the next step is a function of the symmetry of the split, as well as of local elastic stress in the lattice/graph. If the graph is perfectly symmetrical and the stress of each possible path is equal, a particle will be equally likely to propagate along any of the arcs. If the graph is not symmetrical and the energy/stress is different along each arc, the particle is less likely to propagate along the arc with highest stress/energy, possibly following the Boltzmann equation or similar (see Appendix A and Figure 9).

One of the most appealing aspect of this approach is that it provides an explanation of the double-slit experiment (see discussion of that important subject below). This is also a reasonably natural approach to take considering the various fruitful approaches of solid-state physics, the Feynman diagrams, Einstein's original work on a statistical formulation of quantum mechanics, and how the flow of an electric current through an infinite symmetric lattice is calculated (see Appendix A). ${ }^{11}$

\footnotetext{
${ }^{11}$ There is another important consideration in relation to probabilities, as explained by Murray Gell-Mann. [17] Probabilities in quantum mechanics only appear
} 
There is another consideration with regards to the elastic stress and expanding Universe. If the Big Bang was indeed what emerged on the other side of a black hole and if the space graph is elastic, it is easy to imagine that the space graph was squished through the bottleneck of a black hole singularity under tremendous stress, similar to a spring compressed to an absolute maximum. After traveling through the black hole bottleneck and emerging on the other end as the Big Bang, the highly compressed space graph would very rapidly unwind and expand (likely changing its symmetry in the process), resulting in something very similar to inflation $[\mathbf{1 8}]$.

Postulate 8. (Nature of mass): The mass of an elementary particle is a measure of the energy added to the lattice (due to the increased elastic stress of the lattice) that results from introducing the corresponding topological defect.

Physical Justification: Given Einstein's equation,

$$
E=m c^{2},
$$

and the assertion that elementary particles are topological defects in an elastic space graph, this appears to be a natural definition of mass.

Corollary: I further propose that the reason for the existence of inertial mass is the elastic resistance of the space lattice against the movement of mass (represented as topological defects in the lattice). This resistance is otherwise known as the Higgs field.

Physical Justification: Let's consider the implications for inertial mass. In the case of a linear non-rotational movement in an arbitrary direction, once you consider the sequence and the set of all elementary graph operations that must occur in order for a body to move in that direction, it becomes clear that in order to execute that movement the space graph has to bend elastically. The same is true for a rotational movement at an arbitrary angle. Since the graph is discrete, for an approximately round flat disc of radius $\mathrm{R}$ the minimal angle it can rotate at is:

$$
\Phi=L_{p} / R
$$

where $L_{p}$ is the Planck length and $L_{p}<<R$. However, if one tries rotating the entire disk at that angle, points that are closer to the

when past histories are sufficiently disentangled from each other through coarse graining and sum over non-essential variables. For fine-grained entangled histories, probabilities cannot be defined because they do not sum to 1 . Instead, a different, more general quantity, D, is utilized instead of probabilities. I have not yet fully considered the implications of that but would like to suggest it as a fruitful direction for further research. 
disc's center (at a smaller distance $r$ from the center) cannot rotate at that angle, since the Planck length is fixed and finite; the smallest angle they can rotate at would be:

$$
\Theta=L_{p} / r \text {. }
$$

Since $\Phi \neq \Theta$, either the space graph, the disk, or both have to bend elastically in order to execute such rotation, with the elastic stress and resistance producing the inertial mass effect. The same is true for an arbitrary angle rotation, following the same logic.

Therefore, the concept of mass arises naturally from the elastic properties, symmetry, and structure of the space lattice (graph). This interpretation of inertial mass is similar if not identical to the definition of the Higgs field and Higgs boson. Within the framework of the Standard Model, elementary (not composite) particles that have mass (except neutrinos) acquire that mass in the process of interacting with a Higgs boson, a process that some physicists have likened to a person trying to move through a crowded room full of people. [19]

In the framework proposed in this article, essentially the Higgs field is the elastic lattice of space, and the Higgs boson is the elastic distortion of that lattice. Particles acquire mass due to the elastic distortion of the space lattice: the lattice has to bend for a topological defect to come into existence, and the lattice has to bend again for a particle (topological defect) to move around. This is the origin of mass. It also makes good sense that the ideas for Higgs boson and for this geometric discrete unified theory both came from solid state physics.

Interestingly, since the space graph is elastic and discrete, there has to be a minimum amount of force that results in movement, because, for anything to move, it has to overcome the adjacent elastic energy barrier. A weaker force than that won't have enough strength to cause an elementary space graph switch, and, therefore, the particle wouldn't move.

For gravitational mass the reasoning is also straightforward: topological defect in elastic space lattice forces the lattice to curve, resulting in gravitational force.

A traditional interpretation of general relativity is that "mass tells space how to curve, and space tells mass how to move." [20] In the proposed framework, our interpretation is: mass is nothing but discrete curved (distorted) space! A curved (distorted) space lattice IS mass! This is a deep and profoundly simple concept, which satisfies the rule of Occam's razor - entities should not be multiplied unnecessarily. In our framework, the only thing that is assumed and fundamental is the space graph and its cellular automata-like rules of operation. Everything else, 
including elementary particles, all physical 'fundamental' forces, space structure, dimensionality, mass, distance, and quantum numbers are simply emerging properties of the underlying space graph.

Furthermore, in our framework, all known physical forces (e.g., weak, strong, electromagnetic, gravitational) have exactly the same source: the elasticity of the underlying space graph. This is how I propose to achieve unification. ${ }^{12}$

Zero mass particles consideration: What about the particles that have zero rest mass such as photons and gluons? Our working hypothesis is that they represent defects that cannot topologically be static, since this would result in a graph configuration that is topologically prohibited. It is perhaps similar to a falling stack of dominoes with each falling domino knocking over the next one, creating an everspreading dynamic wave.

2.2. Additional postulate needed: Conservation Laws. It appears that appropriate discrete conservation postulates have to be defined on the graph. ${ }^{13}$ Since I do not know the exact functional form of these conservation postulates, I will refrain from formulating them as a definite list. However, I would like to propose several possible candidates (for an isolated system): 14

- The total number of arcs of the graph may be conserved

- The total number of nodes of the graph may be conserved

- A discrete generalized version of the energy stress momentum tensor may be conserved

\footnotetext{
${ }^{12}$ The observable difference between the four fundamental forces results from specific local geometries of the space graph and specific local defects (particles) involved. But, as the symmetry of the space graph increases (perhaps inside a black hole or at the beginning of the Big Bang), at the highest symmetry state, all four forces will merge into one.

${ }^{13}$ A plausible alternative to conservation postulates would be to explicitly list all allowable operations on the graph (or, equivalently, all prohibited operations) in the style of generalized cellular automata. If an appropriate set of allowed operations is chosen, the conservation laws would be a result of the collective properties of this set of allowable operations. In a way, this is a logical duality: one can either define conservation laws and deduce the corresponding allowable operations, or one can define the allowable operations and deduce the corresponding conservation laws.

${ }^{14}$ In a continuous space, Noether's theorem [21] relates symmetries to conservation laws. There already exists a number of published papers on expanding Noether's theorem to discrete lattices, including but not limited to an article by Wendlandt et al.. [22] Those approaches may help derive exact conservation laws and space lattice symmetries for discrete models of the Universe such as our proposed framework.
} 
- The total information content of an isolated system is conserved.

\section{Discussion}

3.1. Guiding Considerations of the Proposed Theory. The following guiding principles and considerations have been a driving force for me in the development of this theory. In my view, any variable that occurs in valid equations of theoretical physics must have a very specific and tangible physical meaning and should be taken literally whenever possible. For example, Planck has originally considered his quants a mere calculational trick rather than physically existing objects. Einstein instead took them literally as physical objects and, in doing so, laid down the foundation of quantum mechanics, as well as explained the photoelectric effect.

Conventional interpretation of Schrodinger's equation and the wave function is that the square of the amplitude of the wave function is a probability density. In my view, probability is not a tangible physical quantity; even less so is the square root of a probability density. Therefore, conventional interpretation of quantum mechanics misrepresents the true nature of the wave function. Assuming that Schrodinger's equation is correct, at least to a high degree of approximation in continuous space limit, I therefore must treat the wave function as a representation of a physical object. In the proposed theory described in this article, the wave function has a very specific physical interpretation. It is a measure of mechanical/elastic stress caused by distortion in the underlying space graph. Unlike the square root of probability, stress and distortion have straightforward physical meanings.

Furthermore, Schrodinger's equation and the wave function incorporate complex numbers in the formulas, without necessarily explaining what complex numbers physically mean. But what is the physical meaning of a complex number? In mathematics, complex numbers are interpreted through introducing an additional dimension (i), together with appropriate commutation, addition, and multiplication rules. Therefore, if Schrodinger's equation is to be taken seriously as it should, the presence of complex numbers in these equations indicates that our Universe has an additional dimension described by the imaginary part of the wave function. This dimension must physically exist with slightly different commutation, addition, and multiplication

rules (as compared to real numbers) that follow the algebra of complex numbers. 
Another hint that this interpretation is on the right track is the Kaluza-Klein theory. [23] Although the theory was eventually discarded, the fact that Kaluza was able to derive both Maxwell's equations and the equations of general relativity from a single unified framework by assuming the existence of a fourth dimension that is bound in a tight circle is deeply profound, as well as puzzling and mysterious. I do not believe that this was a mere coincidence. Even if Kaluza's formulas did not correctly account for all of observable physics, he must have been at least partially on the right track, and it may be quite worthwhile to try to reproduce the Kaluza-Klein theory on the discrete space graph framework proposed in this article. It appears plausible that our Universe may indeed have 4 discrete spatial dimensions, with the fourth dimension corresponding to the imaginary part of the wave function and bound in a circle as proposed by Kaluza-Klein.

Another argument along the same lines is the explanation of the quantum tunneling effect. If one assumes extra spatial dimensions (as described in the paragraph above) or non-local links as described in Postulate 4, the tunneling effect explanation becomes clear. Instead of going through the energy barrier, a tunneling particle goes around the barrier (see details below).

The presence of Planck distance and Planck time in the equations of theoretical physics suggests that the Universe may be discrete at its most basic level. If so, a discrete graph appears to be a natural framework for modeling the Universe. General relativity must be correct on the macroscopic level because of its profound elegance and consistent experimental confirmation. If so, a discrete version of general relativity would be a space graph with dimensions that fit our observations, and an elementary unit of space curvature would be an elementary local discrete topological distortion of that space graph.

\subsection{Qualitative Explanation of Important Physical Phe-} nomena. In this section, I propose and discuss a qualitative explanation of important physical phenomena, concepts, and equations utilizing the framework of the space graph described in this article. It is interesting to note that a number of puzzling phenomena, for example quantum entanglement, have a natural and compelling explanation within the framework.

3.2.1. Wave Function Nature and Wave-Particle Dualism. The dual wave-particle nature of elementary particles is explained as follows. The physical meaning of a wave function corresponds to a degree of elastic stress in the underlying space lattice. The elastic stress field 


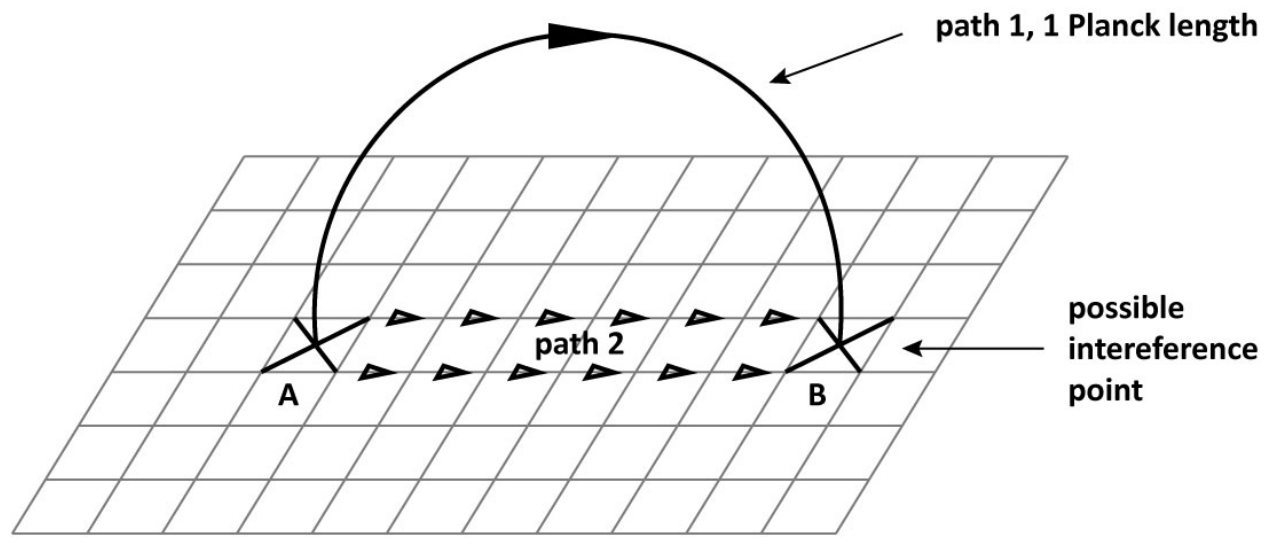

FiguRE 10. Propagation of signal between entangled particles can follow "fast \& direct" 1-Planck-length path. It should take 1 Planck time interval for the signal to propagate between entangled particles following the short path. Interference between the waves traveling through the short path and long path is also possible, although unlikely for most configurations due to the vast differences in distance ratios between a short path and long path. The changes might also propagate instantaneously between $\mathrm{A}$ and $\mathrm{B}$ due to topological conservation laws on the lattice.

created in the space lattice by a topological defect that IS an elementary particle (see Postulates 4 and 5 above) is responsible for the wavelike properties of a particle, while the topological defect at the core of the particle is responsible for its particle-like properties. Stress and distortion waves, caused by topological defects (elementary particles), propagate through the lattice in a distributed manner analogous to mechanical stress waves, which can produce wave interference effects, while the topological defect (the center and 'heart' of an elementary particle) has a specific location within the graph/lattice and behaves like a particle. Because a topological defect causes an associated distributed elastic stress field (wave) to exist, wave-like and particle-like properties of elementary particles are always coupled with each other.

3.2.2. Quantum Entanglement. Entangled particles are simply connected through a direct graph link as illustrated in the Figure $4 \mathrm{C}$. This completely resolves the apparent non-locality and Bell Theorem contradictions.

The so-called "spooky action at a distance" that troubled Einstein so much is simply explained by the fact that entangled particles are directly connected by a graph's arc (a discrete quantum version of the Riemann fold puncture). Even though the entangled particles appear to be far away from each other, the presence of a direct spatial link between them on the order of one Planck length (see path 1 in Figure 
10) enables their respective states to affect each other instantaneously or almost instantaneously. Therefore, quantum entanglement is not an 'action at a distance'; it is, in fact, a local action due to the presence of an arc (one Planck length in size) that directly connects the entangled particles.

Although traditional quantum mechanics postulates that entangled particles affect each other instantaneously, the proposed framework appears to suggest that there may be a delay of 1 Planck time to allow propagation of the signal from one particle to another through one Planck length. Although 1 Planck time is an extremely brief duration, it is nevertheless greater than zero. It would be interesting to see whether or not a delay of one Planck time in entangled particles interactions occurs in our Universe, and if so, what would be the consequences.

Another possibility is that due to conservation laws of the space graph (see Additional Conservation Postulate above) both of the entangled particles may have to change instantaneously and simultaneously as part of a single operation. In that case there will be no delay at all.

In summary, entangled particles are connected through a direct space graph link. This explains the so-called "spooky action at a distance," as well as the non-local paradoxes of Bell's theorem.

3.2.3. Wave Function Collapse. The collapse of the wave function is the process of topological transformation or breaking of the topological defect/knot representing an elementary particle. The local stress produced by the topological defect disappears because the knot/defect snaps, releasing the elastic stress through rearrangement of the connections (arcs, edges) between the graph's nodes. Therefore, the wavelike long-range stress in the space lattice also gradually relaxes to zero through the propagation of stress waves (which are presumably spreading at the speed of light in our universe). During this process, the information represented by the knot leaks into the environment, following conservation laws. In this context, information is an irreducible quantitative description of the space graph geometry/topology and dynamics, essentially a digital encoding of the graph configuration in the sense of information theory and Shannon's formula. [24]

A snap in the space graph would most likely occur in the location of the highest stress (with the highest probability), but it might also occur in less stressed areas of the graph (albeit with a lower probability). The probability of a snap in a particular location is proportional to the square of the amplitude of the elastic stress in that location (as illustrated by the stick-bending analogy in the Physical Justification 
(a)

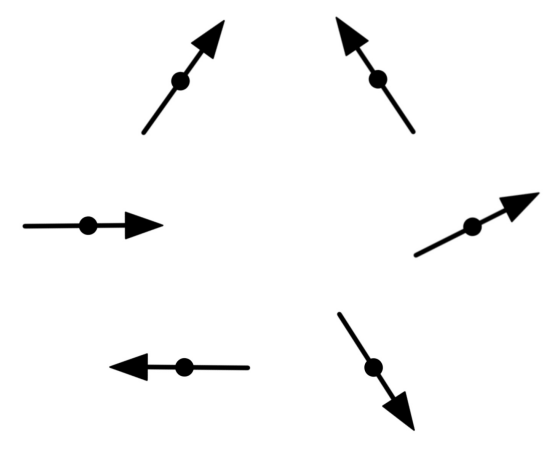

(b)

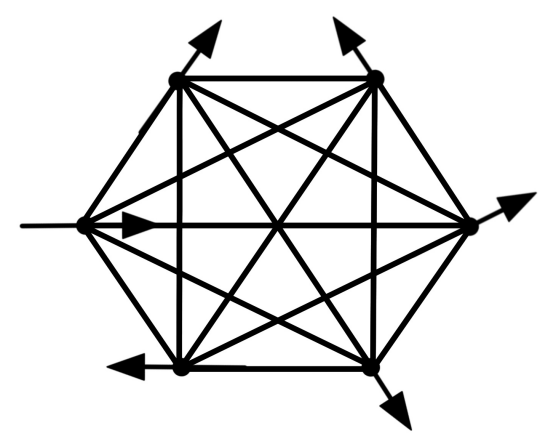

Figure 11. Degrees of freedom in classical and quantum systems. A: The number of degrees of freedom in a classical system is proportional to the number of particles. B: The number of degrees of freedom in a quantum system is a tensor product of the degrees of freedom of individual particles due to the possibility of entanglement between particles. Entanglement is explained as direct spatial links of one Planck length between the particles on the space graph.

discussion of Postulate 7 above), which is why the probability density of finding a particle in a specific location classically is proportional to the square of the amplitude of the wave function.

3.2.4. Degrees of freedom in a multi-particle system. One of the strange aspects of quantum mechanics is why the number of degrees of freedom in a multi-particle quantum system grows exponentially faster than the number of particles, rather than always being proportional to the number of particles (as it would in a classical system). In the proposed framework, the answer for this is straightforward. The extra degrees of freedom describe the presence (or absence) of direct spatial links between the particles, the properties of these links, such as elastic stress level, and the topological angles between the orientations of the different particles (see Postulate 2 and 3 and Figure 11).

Furthermore, for a system of $\mathrm{N}$ quantum particles, the reason why the number of degrees of freedom grows geometrically rather than as $N^{2}$ is that the degrees of freedom describe the configuration space (a tensor product), where the degrees of freedom multiply rather than add. The reason for why the configuration space is a tensor product is inherently in the possibility of direct spatial links between the particles, meaning entanglement features. This point is well illustrated in the article by T-D Bradley, which says: "The tensor product captures all 
ways that basic things can interact with each other." [25] Indeed, in the framework of the proposed theory the possibility of direct spatial links between all particles (in other words, possibility of entanglement) indeed captures all ways that elementary particles can interact with each other.

3.2.5. Pathway to the unification of four fundamental forces. The approach presented here asserts that all four known fundamental physical forces are interpreted as four different types of elastic stress patterns caused by different types of topological defects within a discrete space graph, as described above. The exact details of geometry and topology of those four distortion types have yet to be discovered, as well as the local symmetry of the structure of the proposed space graph, and the structure of specific topological defects representing elementary particles in our Universe. This will be a challenging area of further research for us. ${ }^{15}$

3.2.6. The Big Bang. In the framework of the proposed space graph theory, the Big Bang is interpreted and represented as a phase transition of the space graph, similar to how a chilled liquid freezes or how crystals grow out of a concentrated solution.

Current theories suggest that the four fundamental forces exist as a result of a series of stepwise breaks in symmetry. Using a similar approach, I assume that initially the proposed space graph at the moment of the Big Bang stage was highly symmetric, resulting in the unification of all four fundamental forces. Presumably, the graph then

\footnotetext{
${ }^{15}$ Speaking of simulations, Stephen Wolfram was rumored to run a powerful computer in his basement searching for the exact cellular automata configuration that would reproduce our Universe. Perhaps, a similar computerized search approach should be tried for the space graph theory proposed in this article. Perhaps those calculations could be carried out in parallel batches on GPU hardware, hopefully revealing a substantial increase in performance compared to execution on the CPU. Or perhaps the calculations could be distributed over a network of computers, whether CPU- or GPU-based, similar to SETI, leveraging scale to achieve increased performance. Such calculations may be carried out step-by-step by iteratively going through all known local symmetry groups, starting with the reasonably simple ones, deriving a list of allowed and reasonably simple topological defects within each group, and iterating through that list, defining either a set of lists of allowable operations or a set of conservation laws for each symmetry group, defining elastic properties of the graph, calculating through the list of possible particle interaction iteratively, and checking whether the results match experimental and observational data. A SETI-like program can be made general enough to run on both CPUs and GPUs to ensure maximum scale; SETI, for example, currently supports both CPUs and GPUs. A similar large-scale program can be written and distributed broadly to enlist users to donate some of their spare computing resources to the quest focused on discovering the most fundamental physical laws of the Universe.
} 

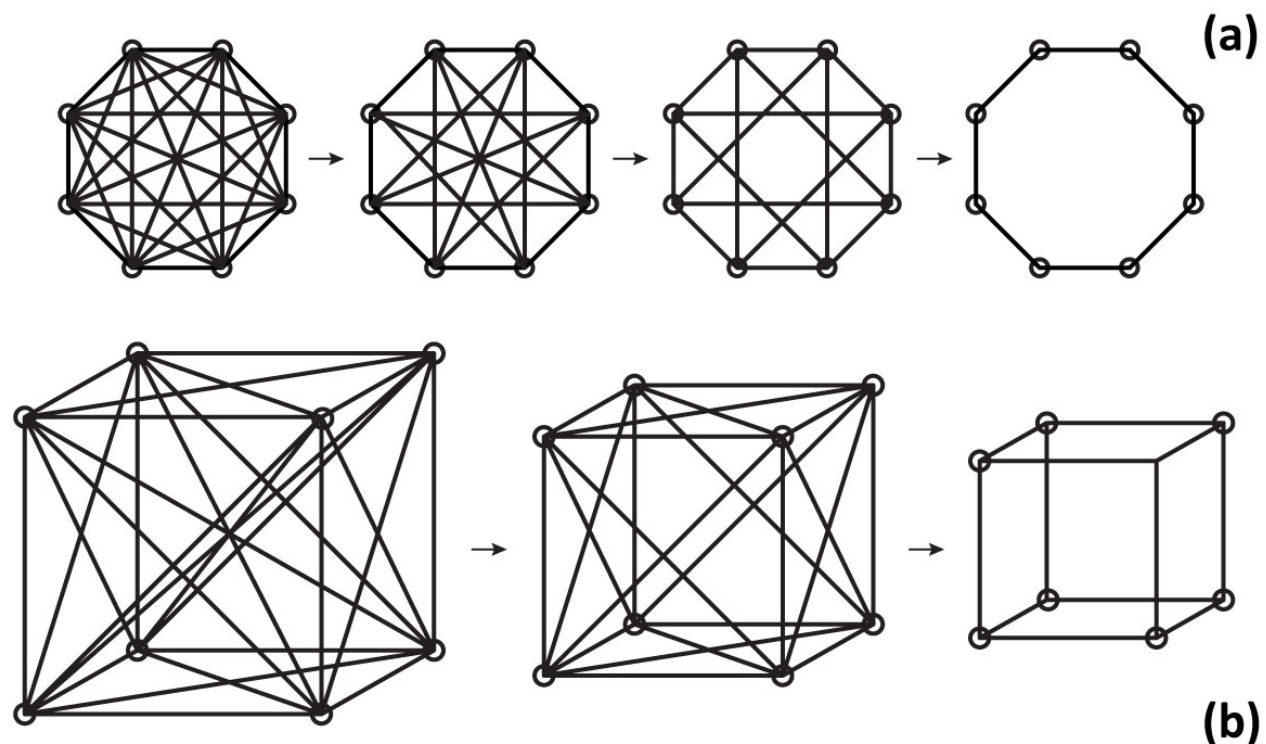

FIgURE 12. Step-wise symmetry breaking on two small graphs. B may indeed resemble the discrete symmetry breaking process that our Universe went through right after the Big Bang, although it appears more likely that our Universe microscopic symmetry incorporates tetrahedral rather than cubic symmetry (see discussion below).

went through three sequential phase transitions with each transition resulting in less symmetry and producing a new split between the previously unified fundamental forces. After three subsequent breaks in symmetry, the Universe ended up with the four fundamental forces as we know them today.

Figure 12 illustrates two possible scenarios of symmetry breaking on small graphs with each transition resulting in a lower symmetry state. The end state in Figure 12B may be mapped to the three-dimensional structure of a cube which resembles the three-dimensional space of our universe. I do not mean to assert that the actual symmetry groups of our universe are exactly represented by these images; I merely wanted to illustrate the concept of symmetry breaking of the space graph. In fact, our best guess of the true symmetry of our Universe is a triangularbased symmetry (for instance, tetrahedral), as proposed below in this paper.

3.2.7. Black Holes. Similar to the Big Bang (see above), within the proposed framework of the space graph, black holes represent a specific topological phase state of the space graph that is different and distinct from "normal" space. Since black holes appear highly symmetric, at least on the outside boundary, ("black holes have no hair"), I think 
that the symmetry of the space graph inside a black hole is higher than outside.

That supports the hypothesis suggested by multiple researchers over time that the Big Bang is what emerged on the other side of a black hole. If we also assume that complete time reversal symmetry plays out in that scenario, since the Big Bang underwent three subsequent breaks in symmetry, I propose that inside a black hole three subsequent symmetry unifications take place step-wise as one gets closer to the center (although it would probably depend on specific stress and energy values along the trajectory inside the black hole, similar to how that works in phase diagrams of regular matter states).

3.2.8. Interactions between elementary particles. Topological defects of the space graph (elementary particles) may recombine, sometimes resulting in new particles or in annihilation (see Postulate 4 and Figure 3 ), subject to conservation laws (Postulate 7), while preserving certain geometric and topological invariants of the space graph (e.g., the sum of the quantum numbers of particles for each distinct type of quantum number).

In addition to the local transformations that occur when particles meet and transform through a rearrangement of arcs and/or nodes in the space graph (transmutation), particles may also affect each other at a distance $^{16}$ (but not instantaneously, unless directly connected by an arc, as all interactions should move no faster than the speed of light), resulting in scattering or attraction. The mechanics of this interaction is through the elastic stress field of the space graph. This is very similar to how defects in crystalline matter (for example, dislocations in metals) attract or repel each other through the mechanical stress field that they induce within the space lattice. ${ }^{17}$

3.2.9. Quantum Tunneling. In the quantum tunneling effect, a particle is assumed to go through the energy barrier. In our framework, I propose that the particle actually goes around the barrier. The process of 'going around' may either happen step-wise alongside (around) the energy barrier or in one long jump of one Planck length around the barrier (see Figure 13 for a graphical illustration).

\footnotetext{
${ }^{16}$ Here I use the words 'location' and 'distance' in the graph sense

${ }^{17} \mathrm{~A}$ similar process is also known to occur for magnetic dislocations, although, in this case, the cause is the electromagnetic field rather than the elastic mechanical stress field.
} 


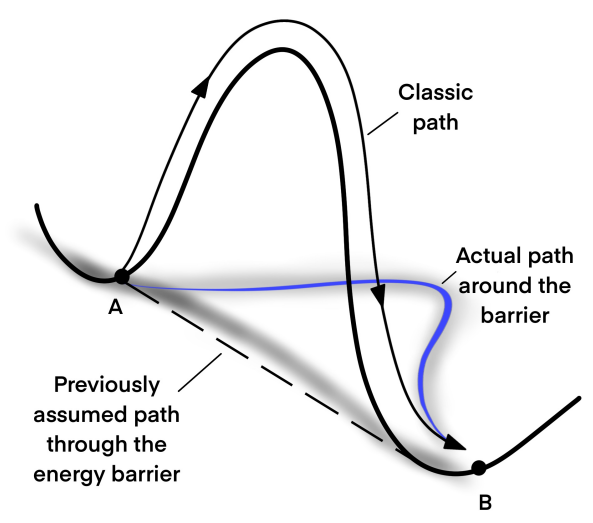

Figure 13. Explanation of quantum tunneling effect: particle goes around the energy barrier into the fourth dimension.

3.2.10. Interpretation of Strings Theories. The Big Bang was a highly dynamic process, producing our Universe as a consequence. Furthermore, it had been known for a while that our Universe is expanding, and it has been recently proven that the expansion is accelerating. $[\mathbf{2 6}, \mathbf{2 7}]$ These findings suggest that there exists a physical process responsible for the creation of space. Therefore, any truly fundamental physical theory must explain how space is created and cannot merely assume that space is just a background. For this reason, string theories in any form are unlikely to be the final answer because they take space for granted as a background. This point has been previously emphasized by many quantum gravity researchers, including Lee Smolin and others. [28]

There exist two distinct possibilities as to how to interpret strings within the framework of the proposed space graph. At the moment, I am not taking a definite position on which one of these two possibilities is actualized in our Universe; this will be an area of further research. The possibilities are:

- Option 1: Strings can be interpreted as discrete imaginary contours (either open or closed) or branes on the underlying discrete space graph proposed in this article. They do not correspond to any physically existing objects, aside from the fact that those imaginary contours can be arbitrarily chosen within 
A

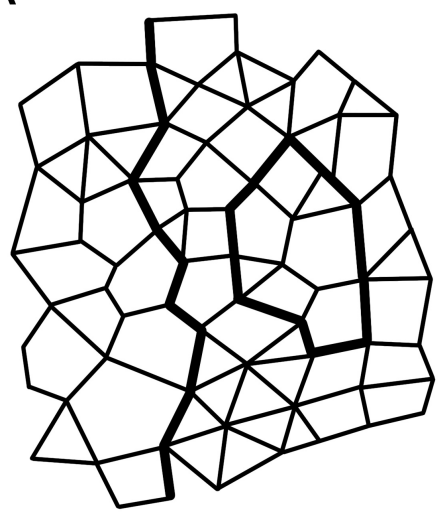

B

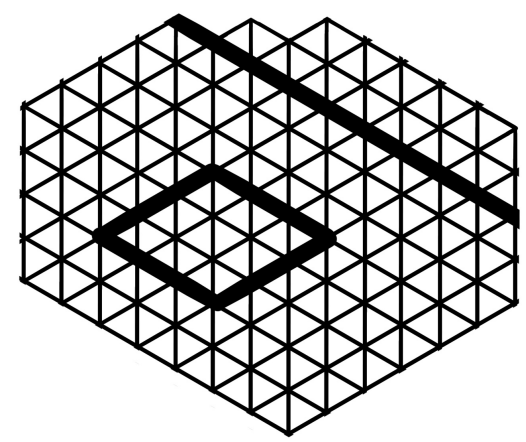

FIGURE 14. Plausible interpretation of strings: imaginary contours on the discrete space lattice. One open string and one closed string are shown here on an irregular space lattice (A) and a regular space lattice (B).

the space graph and follow the edges of the space graph. The specific string lines can be drawn in any arbitrary fashion as long as they follow the space graph's surface and edges. The choice of a specific contour is arbitrary and the only rule is that the contour is a part (subset) of the graph. As such, strings are merely a made-up construct similar to the discrete elements approach utilized in civil engineering models of stress analysis. They have to follow the topology and geometry of the underlying space lattice just like the network of discrete finite elements representing a building has to match the topology and geometry of the building in civil engineering models. (Figure 14) offers a visual illustration. (Figure 14A) displays two imaginary strings (one open and one closed, highlighted by thick lines) within an irregular space graph, while (Figure 14B) does the same on a regular graph.

Even if strings are completely imaginary objects, they may still offer calculational benefits. First, they encode the topology and geometry of the space graph as they have to follow the graph's surface. Second, because they are extended objects, they may help avoid singularities in our calculations, just like in the calculus of complex numbers, where one can calculate an integral over a singularity by following a closed contour path integral around the singularity. Third, as strings are usually 
thought of as elastic objects, they might also be utilized to represent the elastic properties of the underlying space graph for computational purposes.

There is yet another interesting take on the M-theory of strings along these same lines. One can consider all arcs (and combinations of arcs) in the space graph to be strings, which have elastic properties as described above (and as described by equations from popular string theories). Any connected contour or line one draws on the space graph along the edges can be considered a string. Therefore, the entire space graph can be viewed as being interwoven from a set of strings. There is one important difference from traditional strings theories; specifically, if one is inclined to view the space graph as an ensemble of strings, the strings do not exist in space. Instead, they represent space. Space is nothing but an ensemble of strings appropriately woven together (chosen arbitrarily as long as they cover the entire graph and follow the graph's arcs). Conceptually, this is just another alternative way to describe the space graph. Perhaps this is the ultimate description and explanation of the M-theory of strings.

- Option 2: Alternatively, another possibility is that strings could also be interpreted as physically existing extended topological defects within the underlying discrete space graph, similar to dislocations in crystals (see Figure 5). In this case, the energy of a dislocation would be (at least to a first order of approximation) proportional to its length (see more on that below), which is similar to some of the energy equations derived from string theory.

3.2.11. Quarks. For a one-dimensional dislocation in solid state physics, its energy is generally proportional to its length. (If a dislocation is bent, there may be second-order energy terms depending on the specific geometry). It appears plausible to suggest that quarks are topological defects that must be connected for geometrical and topological reasons by a one-dimensional space graph dislocation. This would explain why it is so hard to separate quarks and why the energy of such a system would grow linearly with distance.

3.2.12. Magnetic Monopoles. Perhaps the reason that no one has ever observed magnetic monopoles is that the corresponding topological defect structure would require adding an infinite amount of mechanical stress energy to the space lattice if one tries to calculate an integral over the entire lattice. 


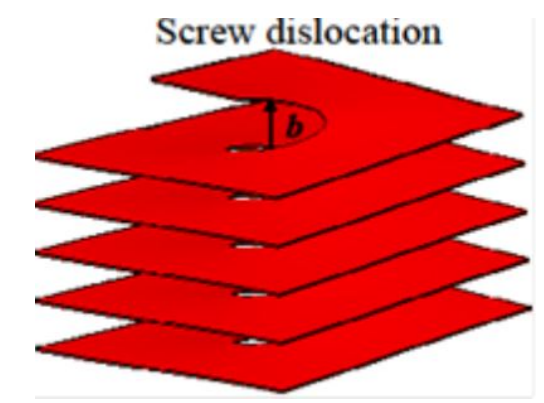

FiguRE 15. Screw dislocation in a regular 3D lattice has an uncanny resemblance to an electromagnetic wave. Image credit: http://www.crpp-bordeaux.cnrs.fr/spip.php?article1324\&lang=en

3.2.13. Screw Dislocations. The geometric structure of a screw dislocation has a certain resemblance to an electromagnetic wave (see Figure 15). Further research will explore whether that resemblance is merely superficial or whether there is indeed a deeper physical connection, perhaps even an identity, between screw dislocations in the space graph and electromagnetism.

3.2.14. Singularities. Since I am proposing a discrete spatial structure with a certain minimum unit of length (Plank length), I expect that no singularities would be predicted by our theory, not even in the center of a black hole. This is analogous to how singularities disappear in string theory as a result of a non-zero string length. Singularities seem to arise when one allows the size of a physical object to become zero or makes other unrealistic "absolute" assumptions, such as absolutely hard objects, etc. Einstein postulated that nothing moves faster than light in a vacuum. Similarly, I assert that nothing can get smaller than the Planck length, which I believe will eliminate singularities.

3.2.15. Calculation of a Black Hole's Entropy. A credible theory in physics must explain observable physical phenomena and calculate their properties in a way that matches experimental results. Since I do not know yet the specific space graph geometry or the mapping of specific geometric defects of the space graph to real-world elementary particles, the scope of calculations I can carry out and compare to reality is limited. Nevertheless, there are certain things I can already calculate utilizing the proposed framework. Specifically, I can calculate the entropy of a black hole and arrive at a correct functional form. Since I assert that the Planck length is the minimum size allowed in the Universe (being the size of an elementary arc of the space graph) and that the space lattice has a regular structure, the number of elementary objects one can fit on the surface of the event horizon of a black hole 
(a)

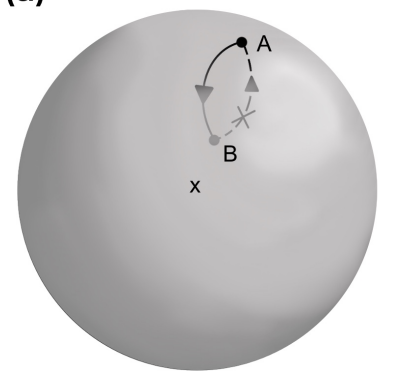

(b)

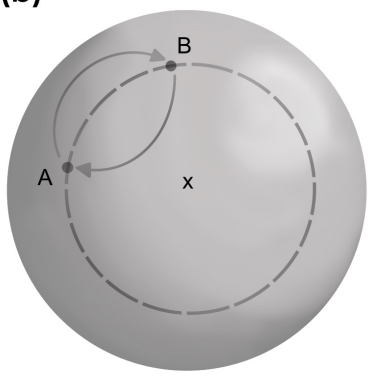

(c)

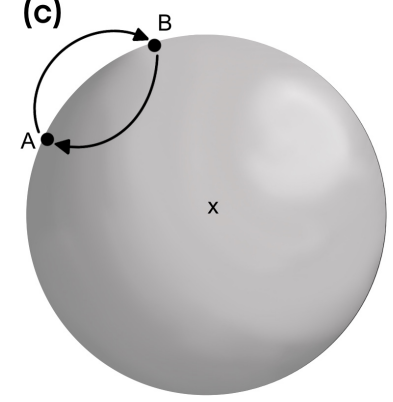

FIgURE 16. Qualitative calculation of a black hole entropy. A: That transposition operation is physically prohibited since nothing can escape a black hole, and therefore it should not count towards black hole entropy. B: Objects inside black hole are not in thermodynamic equilibrium with the objects outside of black hole, and therefore this transposition should not count towards black hole entropy. C: Only transpositions between the objects located right on the event horizon should count towards black hole entropy. Since Planck length and Plank area are finite, and the smallest physically existing object should have a cross-section on the order of one square Planck length, therefore total black hole entropy must be proportional to its surface and be a function of how many elementary Planck areas can fit on the surface of the event horizon.

is proportional to the surface area of the event horizon divided by the square of Planck length. Objects inside a black hole cannot contribute to its entropy because they are not in thermodynamic relationship with the space outside the event horizon and cannot interact with it. Therefore, only the objects on the surface of the event horizon can contribute to its entropy (see Figure 16), and therefore black hole entropy must be proportional to its surface area. This reasoning is practically identical to the one published by Carlo Rovelli in 1996. [29] ${ }^{18}$

The entropy of a black hole can also be calculated using other approaches, as demonstrated by Stephen Hawking, Jacob Beckenstein, and others. [30] However, within the framework proposed in this paper, the number of elementary space graph nodes on the event horizon of a black hole should depend on the local symmetry group and the geometry of the graph, because different local symmetries would result in different packing densities. Hawking has shown that the proportionality coefficient in the black hole entropy formula is 1/4. [31] Further work will focus on calculating the precise local symmetry of the

\footnotetext{
${ }^{18}$ Special thanks to Ian Smith for suggesting the entropy of a black hole as a calculation target.
} 
space graph from that proportionality coefficient proposed by Hawking, Beckenstein, and others, which I hope will help to determine the true symmetry structure of the space graph on the event horizon of a black hole and may also provide ideas as to how to extrapolate the structure and symmetry of the space graph away from the surface of the event horizon and into our "normal" space outside of a black hole.

3.2.16. Interpretation of the double-slit experiment. Feynman once wrote that all the mystery and strangeness of quantum mechanics boils down to the double-slit experiment. [32] Within the space graph framework, the double-slit experiment is explained as follows.

A particle's center (topological defect of the space graph) goes through either one slit or another. However, the expanded space graph elastic stress field caused by the defect is distributed and wave-like in nature. This stress field, being essentially a wave, goes through both slits and creates the interference pattern as a wave should. However, when it is time for the particle to hit the screen, that collision impact forces the wave-particle configuration to "decide" what specific arc in the space graph should break in order to release the elastic stress in the space lattice. Analogous to static mechanics, where a material is most likely to break in the area of greatest stress, the arc of the space graph that is most likely to break and reconfigure will be the one that is under the most stress (see Postulate 5 above). The stress is the largest in the areas where the space graph elastic stress field wave interfered with itself in a way that increased the stress (areas where the constructive interference of the wave is the largest). Therefore, the particle dots will cover the screen most densely in the areas that exactly match the experimentally observed interference pattern.

Once an arc is broken in a specific location, the particle will materialize in that location, but, since quantum numbers and topological and geometric invariants of the graph must be conserved, as suggested above in section "Conservation Postulate Needed," this would cause the topological defect at the heart of the elementary particle to "untie" and cease to exist in all other possible locations. My guess is that the specific mechanism for doing so would most likely be a discrete Riemann fold puncture forming a direct connection between the area where the arc broke and the 'previous' heart of the original topological defect (particle). Another possibility is that this 'defect untie' process would spread through regular space at the speed of light.

If, in the double-slit experiment, one could measure which specific slit the particle traveled through, it would destroy the phase coherence within the wave, and the interference pattern would not emerge on the screen, as seen in numerous experiments. 
I also suggest that the difference between experimental "weak measurements" and "strong measurements" of quantum systems is the following: strong measurements involve breaking (rearranging) the space graph; weak measurements do not cause any space graph rearrangements but they do cause elastic stress fields to interact and affect each other and to convey a certain amount of information in the process of doing so.

\subsection{Open Questions and Future Research Areas.}

3.3.1. Space Graph Geometry. What is the structure and symmetry of the space graph and exactly what type of defect corresponds to each known elementary particle? This is an open question. I would like to mention several possibilities, which are all areas of further research.

One plausible option is that the local space graph symmetry group is triangular, ${ }^{19}$ since this is the simplest discrete symmetry group that can produce an area and volume. It is also one that is the easiest to form statistically in a super-dynamic and chaotic process, such as the Big Bang or the collapse of a black hole. If the probability of an arc or a node in a space graph to be available for forming a bond or a cell in a certain local symmetry configuration is less than 1 , then the probability of finding three such objects to form a triangular cell must be greater than for a quadratic, hexagonal or other cell type (assuming that the underlying probabilities are at least partially independent). Once a triangular cell is formed, other cells are likely to condense on it in the same triangular geometry, similar to the crystallization process in solid state physics, which would presumably result in the structure with the lowest elastic stress and conformational energy. Therefore, a triangular symmetry group appears to be a natural guess.

There is experimental evidence suggesting that the space lattice symmetry group may indeed be triangular-based, or more specifically, tetrahedral. The electric charge of an electron is a multiple of 3 electric charges of the d quark. In the framework of the proposed theory, an electric charge is a local geometrical/topological characteristic of the space lattice and its defects. If those characteristics differ by exactly a

\footnotetext{
${ }^{19}$ Special thanks to Vladimir Mikhalev for collaborating on that insight, as well as on the discussion of rotational symmetry
} 
factor of 3, this is a good indication that indeed the symmetry of the space lattice and its defects is triangular-based! ${ }^{20,21}$

Additional arguments in favor of the symmetry group that incorporates both triangular symmetry and mirror symmetry is the structure of the Standard Model: it contains three families of matter particles, as well as a mapping between electron-muon-tau and corresponding neutrinos, and a similar dual mapping within quarks family. Therefore both triangular symmetry and mirror symmetry are likely to be subsystems of the total discrete space symmetry group.

It is also very important to try to guess correctly the number of dimensions in the spatial graph representing our Universe. That would also establish a base for considering the geometry and topology of defects representing elementary particles. I do not know the true answer in regards to the number of dimensions, but my best guess is that it is 4-dimensional (not considering time, I mean only spatial dimensions here), with the 4 th dimension representing imaginary numbers axis and rolled in a circle. There are two reasons for my guess: (1) imaginary component of wave function in Schrodinger's equation; and (2) Kaluza-Klein theory equations.

Another intriguing, although probably less likely possibility is some higher dimensional generalized form of Penrose tiling or variant tiling. (see Figure 17 and Figure 18) ${ }^{22}$

It also appears plausible, as suggested above, that fermions should be represented by the types of geometric defects that allow only one particle of its kind to occupy a particular location (more than one particle in any one location would presumably result in configurations that are logically impossible or prohibited within the space graph). Bosons, on the other hand, can be represented by the types of defects that allow a limitless number of particles in any one location, without violating

\footnotetext{
${ }^{20}$ In this example, the color and flavors of quarks would naturally be interpreted as certain geometrical and topological characteristics of the space lattice defects that correspond to quarks. The same is true for gluons.

${ }^{21}$ It is important to note that some quantum numbers of elementary particles, specifically spin, differ from each other by a factor of two for certain particles (quarks and leptons have spin 1/2, photon, gluon, W and Z bosons have spin 1). This must also be a consequence of the space lattice symmetry and particle geometry within the graph. That may indicate a mirror symmetry, and, in combination with the multiple of 3 for electric charge, as discussed above, a full tetrahedral, a hexagonal or chiral-tetrahedral symmetry for the space lattice and space lattice defects.

${ }^{22}$ Credits for Figure 17 and Figure 18 images: https://en.wikipedia.org/wiki/Penrose_tiling\#/media/File:Penrose_Tiling_(Rhombi).svg and https://en.wikipedia.org/wiki/Penrose_tiling\#/media/File:Variable_penrose_tiling.svg
} 


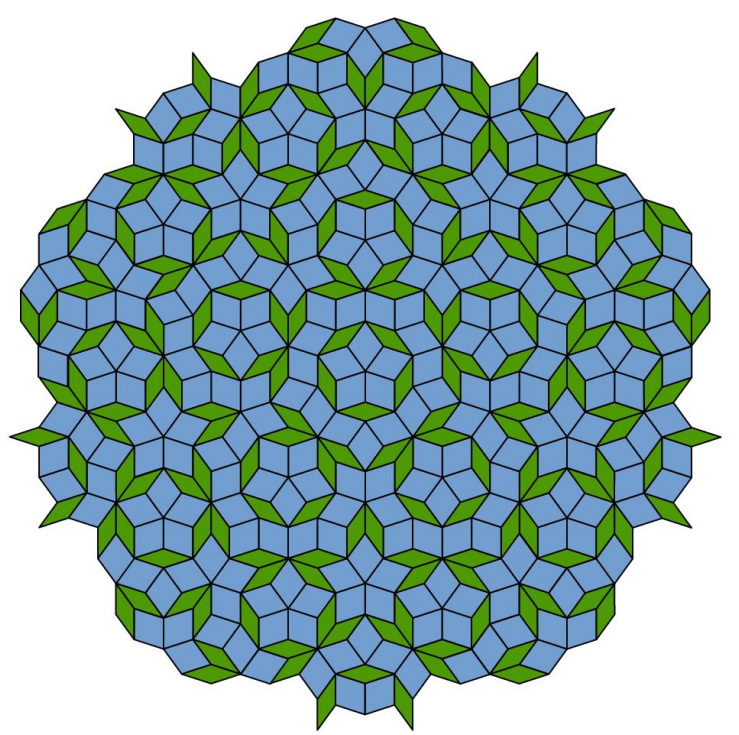

Figure 17. Penrose tiling. It is possible, though not quite likely, that the space graph structure of our Universe is a generalized form of a Penrose tiling for higher-dimensional cases.

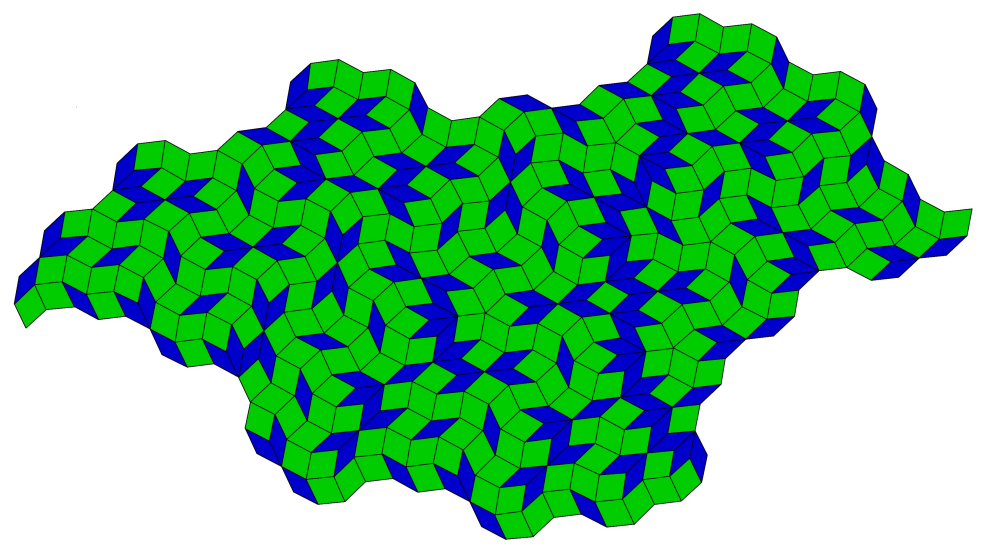

FIgURE 18. Variant tiling: one of generalized forms of Penrose tiling. It is possible, though not quite likely, that the space lattice structure of our Universe is a generalized form of variant tiling for higher-dimensional cases. 
any conservation laws or resulting in any geometric configurations that are logically prohibited by the nature of the space graph.

These questions can be further explored either by analytical approaches or by brute force computation methods on sufficiently powerful computers. Analytical approaches may require insightful guesses and a priori assumptions about the geometries of the space graph and elementary particles (defects), combined with the analytical rigor to carry out the calculations. Perhaps there exists an effective shortcut, such as deriving the geometry of the space graph from the black hole entropy formula (see "Black Hole Entropy" section above). The goal is to either strongly limit the set of configurations considered for computational testing, or perhaps even guess the correct one outright and confirm it through rigorous calculations.

3.3.2. The Paradox of Rotational Symmetry. This issue has caused me a lot of concern in relation to the proposed space graph theory. If the space structure is discrete, how can that be reconciled with the fact that, at least on the classical level in our Universe, we observe seemingly continuous rotational symmetry consistent with SO(3) group? This contradiction seems to grow even larger if one tries to visualize the space graph as a rigid and infinitely regular structure, similar to an ideal crystal in solid state physics. ${ }^{23}$

So, if we assume that, on the smallest scale, the space graph structure is regular, why don't we observe in our Universe a discrete rotational symmetry with fixed angles of rotation (rather than a continuous $\mathrm{SO}(3)$ ), similar to the discrete rotation symmetry group in solid crystals? Some possible explanations may include but are not limited to the following:

\footnotetext{
${ }^{23}$ So far, I have primarily discussed the simplest form of a space graph where all nodes are identical, aside from a variable number of arcs that may be connected to them. However, following the solid-state physics analogy, one can also imagine a graph with more than one distinct type of node, such as a salt crystal, where some nodes represent $\mathrm{Na}+$ and some nodes represent $\mathrm{Cl}-$, as opposed to a diamond crystal where all nodes are the same. Also, in the simplest case, we may assume that not only are all nodes identical but also that the number of arcs connected to each node must be exactly the same. Of course, another possibility is that the number of arcs can vary between 0 and some fixed integer number, as it does in chemistry for chemical bonds between atoms. Finally, we must also consider the possibility of a directed graph with some arcs having a preferred direction, perhaps explaining one-way movement towards the center of a black hole. I would nevertheless hope that a simple solution would accurately correspond to our Universe, as often is the case in nature, with all nodes and/or the number of connecting arcs being the same. My preferred direction is to follow Einstein's maxim, "Make things as simple as possible, but not simpler."
} 
(1) Perhaps, on the smallest scale, rotation is discrete, but, on macro scales, rotations get 'averaged' to a practically continuous $\mathrm{SO}(3)$ rotation as a result of quasi-random irregularities in the lattice structure, space grain boundaries between the adjacent small areas of fixed orientation, and/or the elasticity of the space lattice. This is very similar to the small-crystal grain structure in metals where each grain has a fixed orientation of the crystal lattice but is oriented randomly (or quasirandomly) in relation to its neighboring grains. As a result, the entire metal slab exhibits continuous rotationally symmetry on a classical scale due to the random orientation of the grains with respect to each other averaging out uniformly in all directions on a macroscopic scale. Since the Planck length is so incredibly small, on a macroscopic level, the rotations and translations of the space graph may be made to look smooth, giving the illusion of continuous rather than discrete movement, both translational and rotational.

(2) Alternatively, the space graph structure may be similar to the structure of amorphous metals or water, which have shortrange but no long-range order.

(3) Elastic bending of the space graph structure may also play a role, as discussed above after the Postulate 7, with regards to the nature of inertial mass. ${ }^{24}$

One tries to make as few assumptions as possible when building a new theory; however, some of these assumptions might actually make good physical sense if one considers how the space graph could have evolved or came into existence. In solid state physics, large crystals are rare in nature because arranging elementary nodes (atoms in the case of solid-state physics) in a perfectly regular solid structure with an infinite long-range order usually takes a long time and requires ensuring reasonably stable conditions, such as a fairly fixed temperature, pressure, and chemical composition of the surrounding media during that time period. In nature, crystals typically form either when the temperature drops and a liquid freezes into a solid (for example, when melted iron cools down and solidifies or water freezes into ice) or through a slow process of chemical deposition from a concentrated solution, such as salt crystals forming from an over-saturated brine. Furthermore, the growth of a crystal typically starts in a location that has some kind

\footnotetext{
${ }^{24}$ Einstein arrived at the idea of curved space by considering the geometry of a rapidly rotating disk. [33]. Perhaps, we also could derive valuable insights by considering the geometry of a rotating body within a discrete space.
} 
of fluctuation or defect (e.g., a random local fluctuation in the concentration of a solvent, a minuscule fluctuation in local temperature, a crack on the wall of the vessel holding the liquid phase of the material). In most cases in nature, the solvent in the liquid phase typically contains many small fluctuations and defects and the walls of the container holding the liquid contain many irregularities. Also, the temperature usually changes quickly enough in most physical processes that involve crystals. As a result, a large number of small crystals typically start to form almost simultaneously in multiple locations and in random or quasi-random orientations. Once the material completely solidifies, it becomes an ensemble of many small crystals with random orientations. For example, metals typically contain a large number of crystalline grains in quasi-random orientations with respect to each other.

In a similar manner, if one assumes that space was created during the Big Bang and/or perhaps that the Big Bang was what emerged on the other side of a black hole, all indications are that this would have been a violent and dynamic process. There are also indications that the Universe rapidly cooled down after the Big Bang. Under these fastmoving and chaotic circumstances, there would not have been enough time for one infinitely uniform regular space graph crystal to evolve through the entire space graph. Instead, the Universe should have plausibly ended up with an extremely large number of small space graph crystals (grains) with different orientations connected at their boundaries, similar to what happens when liquid iron cools rapidly and solidifies.

One should be even more confident of this expectation if we consider the random quantum fluctuations that are an inherent nature of the quantum systems. Quantum fluctuations must have been present in the early universe, as confirmed by studies of cosmic microwave background radiation (CMBR). Fluctuations are needed to seed the origins of multiple crystal grains simultaneously, as seen in solid state physics and physical chemistry. Therefore, quantum fluctuations would have likely resulted in multiple crystalline areas of the space graph growing almost simultaneously in random orientations with respect to each other. Perhaps a more detailed analysis of CMBR may even contain clues to the symmetry structure of the space graph. For instance, perhaps the largest visible universe-size patterns of CMBR is an imprint of the very first elementary graph cell that has formed during the Big Bang? It would also be worthwhile to examine the range of all scales in CMBR.

Finally, if one assumes that the Big Bang was what emerged on the other side of a black hole, matter would have likely fallen into 
the black hole in a chaotic, violent random process, possibly seeding the fluctuations on the other side of the black hole, which became our Universe.

For all these reasons, the small grain-structure of the space graph may indeed be plausible. If we assume a sufficiently small size of the grains within the space graph and a random orientation with respect to each other, we would end up with a Universe that has a discrete rotational and/or translational symmetry on the Planck length scale and a continuous $\mathrm{SO}(3)$ symmetry on the classical scale, such as we observe in our Universe.

Another possibility, as previously mentioned above, is that perhaps the geometry of the space graph is a generalized higher-dimensional version of variant tiling (see Figure 18), Penrose tiling ${ }^{25}$ (see Figure 17) [34], Gummelt covering, [35] or a quasi-crystal type of structure. [36] Our understanding of such structures is evolving but much still remains to be learned about them. It must also be noted that the Penrose, variant, and Gummelt tilings and coverings exist in space, while the proposed space graph is space. Therefore, the class of possible structures of interest within the space graph is likely significantly larger than a class of traditional 'in-space' tilings and coverings.

3.3.3. Experimental Verification and Predictions of the Proposed Model. For a physical theory to be credible, it should be verifiable through experimental means. Within the proposed framework, there is a specific prediction that might be verified utilizing current experimental methods.

I have suggested that entangled particles are connected through a direct spatial link of one Planck length (Figure 4C). Consider entangled particles which stop being entangled. At first, the link between the entangled particles breaks. Since an arc has just disappeared, assuming that conservation laws preserve the total number of arcs in a closed

\footnotetext{
${ }^{25}$ What I mean by "generalized" Penrose tilings is the following: (1) Penrose tilings exist in $2 \mathrm{D}$, while we need an equivalent $3 \mathrm{D}$ or $4 \mathrm{D}$ version of it. There exist a number of math papers extending Penrose tilings to higher-dimensional space; and (2) Penrose tilings exist in 2D Euclidean space, while our proposed space lattice is space, and furthermore it can bend elastically, does not have to be Euclidean, the number of local dimensions might change, and it may include dimensions that correspond to the imaginary part of complex numbers in Schrodinger equation (and therefore may have different commutation and multiplication rules). Therefore we essentially need a generalized graph-topological higher-dimensional version of Penrose tilings. The reason I have considered Penrose-style structures in the first place is that they still have a structure that is regular enough so that we can speak about topological defects (elementary particles) within that structure, and they can still cover space fully and without gaps in the Euclidean limit.
} 
(a)

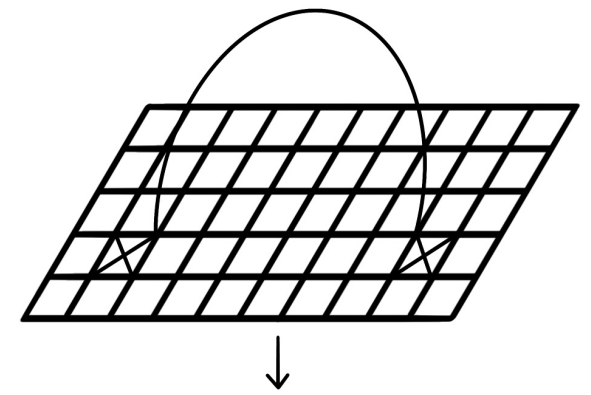

(b)

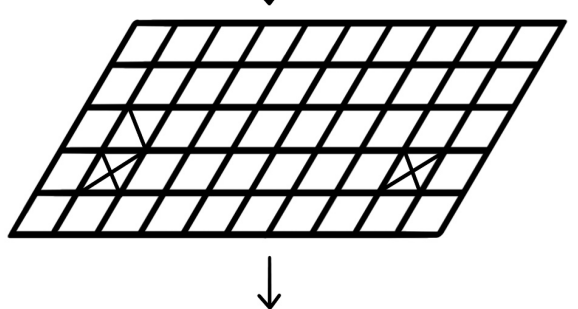

(c)

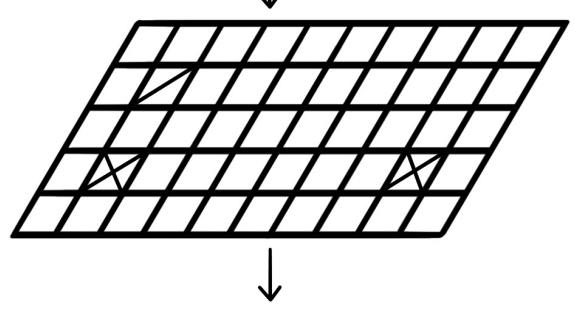

(d)

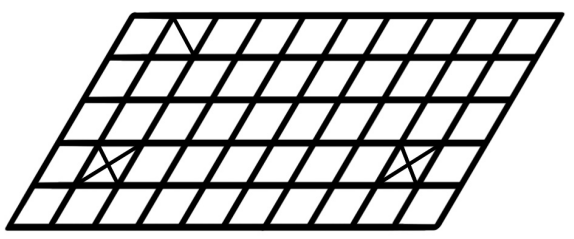

Figure 19. This image illustrates that when entangled particles stop being entangled, a new particle must be emitted. A: represents entangled state: two entangled particles (topological defects of the lattice) are directly connected by a spatial link of one Planck length. B: The entanglement breaks, but assuming that the total number of arc must be conserved due to a conservation law, another arc should appear, likely in an adjacent location. That new arc is also a topological defect, and therefore must represent a newly born emitted particle. C,D: The emitted particle moves further away from the original location. Therefore the proposed discrete geometric theory may be verified experimentally by analyzing whether entanglement breaking results in a new particle emission. If detected, the geometric nature of the emitted particle would also become clear as it must correspond to one extra arc in the space graph. 
system (see the section "Conservation Postulate Needed" above), an extra arc would appear in a topologically adjacent area and perhaps then propagate through the space graph (Figure 19 ). This extra arc would also represent a topological defect, which, by definition, would be an elementary particle. What kind of elementary particle would be represented by an extra arc in the space graph? Since it appears that an extra arc would introduce a relatively light deformation of the space lattice compared to other possible types of topological defects, the particle emitted when entanglement breaks should therefore have a small mass. The most likely candidate would be neutrino (which has a very low but non-zero rest mass). A less likely possibility would be a photon. More speculative candidates include dark mass particles, dark energy, or perhaps a graviton. The antiparticles of these particles are also a possibility.

In summary, a break in entanglement would result in the emission of an elementary particle. With some ingenuity, the emitted particle may be experimentally detected. ${ }^{26,27}$

Another possible experimental verification is the following. Although the Planck length is much much smaller than anything modern experimental technologies can probe directly, we could try to observe the aggregated effects of discrete space composition on particles that travel over large distances. This has been originally suggested in a research paper by Rodolpho Gambini and Jorge Pullin [37]. We can analyze light (or any radiation) arriving to Earth from far-away galaxies billions of light-years away. Over such vast distances, there must be a measurable imprint on that radiation by the structure of space. This signature imprint would be different, depending on whether space is discrete or continuous. We might even be able to derive the exact space geometry and local symmetry on the Planck scale from its imprint on particles of light/radiation traveling over vast distances. We might even be able to detect whether the structure of the space graph is constant throughout the observable Universe.

${ }^{26}$ This idea has been suggested by Alexey Solovey in a private discussion

${ }^{27}$ The suggestion that termination of entanglement connection results in a new particle being emitted would only work if the number of arcs is indeed conserved. But since I am unsure on the exact form of the conservation laws, it is also possible that only the number of nodes is conserved, but not the number of arcs, similar to well-known situations in atomic matter when the number of atoms is conserved but not necessarily the number of chemical bonds. If the number of arcs is not conserved then termination of entanglement would not have to result in particle emission. 


\section{Summary and Conclusions}

(1) This article proposes a unified framework based on a discrete graph model to explain the nature of space and time, the nature of elementary particles, the origin of quantum numbers, wave functions, wave-particle duality, wave function collapse, quantum entanglement, quantum probabilities, degrees of freedom in multi-particle systems and provides an interpretation of quantum tunneling. This framework also explains the origin of mass, fundamental forces, Higgs field and Higgs boson.

(2) An illustrative calculation of the entropy of a black hole is interpreted within the proposed framework.

(3) A new prospective on strings theories and M theory is proposed in the spirit of generalized quantum gravity models.

(4) Cosmological implications of the proposed theory, including the Big Bang and black holes, are explored.

(5) Interpretations of fermions, bosons, and zero rest mass particles are proposed.

(6) Various possibilities for modeling the discrete structure of space are proposed and discussed.

(7) Ideas for experimental verification of the proposed theory are suggested.

(8) Further areas of research include the dynamics and structure of the space graph, identifying the structure of specific geometric and topological defects corresponding to known elementary particles, and elementary operations and conservation laws on the space graph.

\section{Acknowledgments}

Special thanks to Roger Penrose, Brian Greene, and Lee Smolin, whose books inspired me to seek new approaches, and to Vladimir Novikov who taught me foundations of solid state physics. I also would like to express my gratitude to Vladimir Mikhalev, Ian Smith, and Alexey Solovey for their useful discussions, and to Nathan Hageman for helping to edit this paper.

\section{Appendix A}

The purpose of this Appendix is to detail a possible approach for calculating the probability associated with any particular path of a particle through the space graph, which is analogous to calculating the flow of electric current through a conducting lattice. 
Consider an infinite rectangular lattice made of a conducting material, as illustrated in (Figure 9). Assume that each edge of the lattice has an electrical resistance, R. An electric current, I, arrives to node A, and leaves through an adjacent node B (Figure 9A). From this configuration, how can one calculate the aggregate electric resistance of the lattice? I was originally introduced to this problem by the late Dmitri Knizhnik (to whom I am profoundly grateful for being my mentor and an inspiration), and it has the following elegant solution:

First, consider the configuration shown in (Figure 9B) where an electric current, I, arrives to node A and then flows through and out the lattice into infinity (an infinitely remote radially symmetric boundary condition that collects the current, I, back from the lattice). In this configuration, due to the symmetry properties of the lattice configuration, it is clear that each of the four edges that are connected to node A will each carry an equal portion of the current, I/4.

Now consider the configuration in (Figure 9C), where an electric current flows from infinity through the lattice to node B. In this configuration, the resulting aggregate current, I, is being sucked out of the lattice through node B to the outside. Similarly, due to the symmetry of the lattice configuration, it is clear that each of the four edges connected to node B will also carry a current equal to I/4.

According to Kirchhoff's circuit laws, the configuration described in (Figure 9A) can be considered a direct linear superposition of the configurations (Figure 9B) and (Figure 9C). Therefore, the resulting current depicted in (Figure 9A) between the nodes $\mathrm{A}$ and $\mathrm{B}$ should be equal to $\mathrm{I} / 4+\mathrm{I} / 4=\mathrm{I} / 2$, as illustrated in (Figure 9D). The electric resistance of the entire lattice in the configuration shown in (Figure 9A) must therefore be equal to $\mathrm{R} / 2$.

Now, consider what happens if one of the edges in the lattice has a resistance greater than $\mathrm{R}$. In that case, less current would flow through that edge as compared to the configuration when all edges have an equal resistance, $\mathrm{R}$.

I propose that a similar process occurs when an elementary particle (topological defect) propagates through the space graph. If the configuration of the space graph is completely symmetric, the probabilities of a particle/defect following a particular path (arc) adjacent to a specific node are all equal. And if the configuration is not symmetric, then the particle (defect) will be less likely to travel arcs with higher stress.

At the moment, I leave open the functional form of this dynamic equation (or a corresponding cellular automata rule), but I expect that it can be directly derived from the energy function of the space graph, as discussed above in the Postulate 5. 


\section{Appendix B. Publication Note}

This paper has been approximately 20 years in the making. I was originally trained in solid state physics and topology in Russia, and was taught by Sergei P. Novikov, the Fields medal winner, and Dmitri Knizhnik, the author of the Knizhnik-Zamolodchikov equation. I developed most of the ideas presented here during the time period 20002006. Since 2006 I have privately shared my theory with a number of physicists as I was looking to add more mathematical rigor to it, and some of those scientists have since published papers containing similar ideas. This paper contains much less math than I would have preferred because I have not worked in the academia since 1991. I have finally decided to publish my theory as is in the hope to attract colleagues who can help develop this further. Any physicists or mathematicians interested in collaborating please contact me at ybreek@gmail.com. Thank you!

Yan Breek

San Francisco Bay Area, California, March 2020

\section{References}

[1] L. Landau and E. Lifshitz, Quantum Mechanics. New York: Pergamon Press, 1977.

[2] L. Smolin, Three roads to quantum gravity. New York: Basic Books, 2001.

[3] L. Smolin, "Atoms of space and time," Scientific American, vol. 290, no. 1, pp. 66-75, 2004.

[4] R. Penrose, "On the nature of quantum geometry," Magic without Magic, pp. 333-354, 1972.

[5] M. Bojowald, "The semiclassical limit of loop quantum cosmology," Classical and Quantum Gravity, vol. 18, no. 18, p. L109, 2001.

[6] M. Bojowald, "Dynamical initial conditions in quantum cosmology," Physical Review Letters, vol. 87, no. 12, p. 121301, 2001.

[7] M. Bojowald, "Inflation from quantum geometry," Physical Review Letters, vol. 89 , no. 26, p. 261301, 2002.

[8] M. Bojowald, "Isotropic loop quantum cosmology," Classical and Quantum Gravity, vol. 19, no. 10, p. 2717, 2002.

[9] V. P. Frolov, M. Markov, and V. F. Mukhanov, "Through a black hole into a new universe?," Physics Letters B, vol. 216, no. 3-4, pp. 272-276, 1989.

[10] S. Tsujikawa, P. Singh, and R. Maartens, "Loop quantum gravity effects on inflation and the cmb," Classical and Quantum Gravity, vol. 21, no. 24, p. 5767, 2004.

[11] A. Lawrence and E. Martinec, "String field theory in curved spacetime and the resolution of spacelike singularities," Classical and Quantum Gravity, vol. 13, no. 1 , p. $63,1996$.

[12] M. Rees, R. Ruffini, and J. A. Wheeler, "Black holes, gravitational waves and cosmology: an introduction to current research," New York, Gordon and 
Breach, Science Publishers, Inc.(Topics in Astrophysics and Space Physics. Volume 10), 1974. 182 p., 1974.

[13] R. Brandenberger and C. Vafa, "Superstrings in the early universe," Nuclear Physics B, vol. 316, no. 2, pp. 391-410, 1989.

[14] J. A. Wheeler and K. Ford, "Geons," vol. 97, p. 511, January 1995.

[15] S. Wolfram, A new kind of science. Champaign, IL: Wolfram media, 2002.

[16] D. O'Sullivan, "Graph-cellular automata: a generalised discrete urban and regional model," Environment and Planning B: Planning and Design, vol. 28, no. 5, pp. 687-705, 2001.

[17] M. Gell-Mann, The Quark and the Jaguar: Adventures in the Simple and the Complex. Macmillan, 1995.

[18] A. H. Guth, "Inflationary universe: A possible solution to the horizon and flatness problems," Physical Review D, vol. 23, no. 2, p. 347, 1981.

[19] D. Miller, "Politics, solid state and the higgs," 1993.

[20] J. A. Wheeler and K. Ford, "Geons, black holes and quantum foam: A life in physics," 2000.

[21] E. Noether, "Invariante variationsprobleme. nachr. d. könig. gesellsch. d. wiss. zu göttingen, math-phys," Klasse, pp. 235-257, 1918.

[22] J. M. Wendlandt and J. E. Marsden, "Mechanical integrators derived from a discrete variational principle," Physica D, Nonlinear Phenomena, vol. 106, no. 1-3, pp. 223-246, 1997.

[23] T. Kaluza, "Zum unitätsproblem der physik," Sitzungsber. Preuss. Akad. Wiss. Berlin (Math. Phys.), vol. 1921, no. arXiv: 1803.08616, pp. 966-972, 1921.

[24] C. E. Shannon, "A mathematical theory of communication," Bell system technical journal, vol. 27, no. 3, pp. 379-423, 1948.

[25] T.-D. Bradley, "The tensor product, demystified," 2018.

[26] A. G. Riess, A. V. Filippenko, P. Challis, A. Clocchiatti, A. Diercks, P. M. Garnavich, R. L. Gilliland, C. J. Hogan, S. Jha, R. P. Kirshner, et al., "Observational evidence from supernovae for an accelerating universe and a cosmological constant," The Astronomical Journal, vol. 116, no. 3, p. 1009, 1998.

[27] S. Perlmutter, G. Aldering, G. Goldhaber, R. Knop, P. Nugent, P. Castro, S. Deustua, S. Fabbro, A. Goobar, D. Groom, et al., "Measurements of $\omega$ and $\lambda$ from 42 high-redshift supernovae," The Astrophysical Journal, vol. 517, no. 2, p. 565, 1999.

[28] D. Rickles, S. French, and J. T. Saatsi, The structural foundations of quantum gravity. Oxford University Press, 2006.

[29] C. Rovelli, "Black hole entropy from loop quantum gravity," Physical Review Letters, vol. 77, no. 16, p. 3288, 1996.

[30] J. D. Bekenstein, "Black holes and entropy," Physical Review D, vol. 7, no. 8, p. 2333, 1973.

[31] S. Hawking, "Particle creation by black holes," Communications in Mathematical Physics, vol. 46, no. 2, pp. 206-206, 1976.

[32] R. P. Feynman, M. Sands, and R. B. Leighton, The Feynman Lectures on Physics: Quantum Mechanics. III. Addison-Wesley, 1965.

[33] A. Einstein and F. A. Davis, The principle of relativity. Courier Corporation, 2013.

[34] R. Penrose, "The role of aesthetics in pure and applied mathematical research," Bull. Inst. Math. Appl., vol. 10, pp. 266-271, 1974. 
[35] P. Gummelt, "Penrose tilings as coverings of congruent decagons," Geometriae Dedicata, vol. 62, no. 1, pp. 1-17, 1996.

[36] D. Levine and P. J. Steinhardt, "Quasicrystals: a new class of ordered structures," Physical review letters, vol. 53, no. 26, p. 2477, 1984.

[37] R. Gambini and J. Pullin, "Nonstandard optics from quantum space-time," Physical Review D, vol. 59, no. 12, p. 124021, 1999. 